\title{
Multi-agent Logics for Reasoning About Higher-Order Upper and Lower Probabilities
}

\author{
Dragan Doder · Nenad Savić \\ - Zoran Ognjanović
}

Received: date / Accepted: date

\begin{abstract}
We present a propositional and a first-order logic for reasoning about higher-order upper and lower probabilities. We provide sound and complete axiomatizations for the logics and we prove decidability in the propositional case. Furthermore, we show that the introduced logics generalize some existing probability logics.
\end{abstract}

Keywords Probabilistic Logic · Upper and Lower Probabilities - Decidability . Completeness theorem

\section{Introduction}

In the last few decades, uncertain reasoning has become an active topic of investigation for researchers in the fields of computer science, artificial intelligence and cognitive science. The frameworks designed for modeling uncertainty often use probability-based interpretation of knowledge or belief. One particular line of research concerns the formalization of reasoning about probability in terms of logic with a well-defined syntax and semantics [6-10,15,20,22-25].

This work was supported by the SNSF project 200021_165549 Justifications and non-classical reasoning, by the Serbian Ministry of Education and Science through projects ON174026, III44006 and ON174008, and by ANR-11-LABX-0040-CIMI.

This paper contains and extends the results presented at the conferences ECSQARU 2017 [27] and FoIKS 2018 [3].

\section{Doder}

Université Paul Sabatier - CNRS, IRIT

E-mail: dragan.doder@irit.fr

N. Savić

Institute of Computer Science, University of Bern,

E-mail: nenad.savic@inf.unibe.ch

Z. Ognjanović

Mathematical Institute of Serbian Academy of Sciences and Arts,

E-mail: zorano@mi.sanu.ac.rs 
In order to model some situations of interest, where sharp numerical probabilities appear too simple for modeling uncertainty, various imprecise probability models are developed $[2,5,18,21,28,30,31,33]$. Some of those approaches use sets of probability measures instead of one fixed measure, and the uncertainty is represented by two boundaries, called lower probability and upper probability $[13,17]$. Given an arbitrary set $P$ of probability measures, the former one assigns to an event $X$ the infimum of the probabilities assigned to $X$ by the measures in $P$, while the later one returns their supremum.

Those two probability notions were previously formalized in the logic developed in [11], where lower and upper probability operators are applied to propositional formulas, and in [26], where a first-order logic is also considered.

In this paper, we use the papers $[11,26]$ as a starting point and generalize them in a way that we reason not only about lower and upper probabilities an agent assigns to a certain event, but also about her uncertain belief about other agent's imprecise probabilities. Thus, we introduce separate lower and upper probability operators for different agents, and we allow nesting of the operators, similarly as it has been done in [7] in the case of simple probabilities. We first present a propositional variant of this logic, which we denote by ILUPP $^{1}$, and then we extend it to a first-order logic. We prove that ILUPP is decidable and we propose sound and strongly complete axiomatizations for both logics.

Our language contains the upper and lower probability operators $U_{\geq r}^{a}$ and $L_{\geq r}^{a}$, for every agent $a$ and every rational number $r$ from the unit interval (we also introduce the operators with other types of inequalities, like $U_{=r}^{a}$ ). Consider the following example: Suppose that an agent a is planning to visit a city based on the weather reports from several sources, and she decides to take an action if the probability of rain is at most $\frac{1}{10}$, according to all reports she considers. Since she wishes to go together with b, she should be sure with probability at least $\frac{9}{10}$ that $b$ (who might consult different weather reports) has the same conclusion about the possibility of rain. In our language, this situation can be formalized as

$$
U_{\leq \frac{1}{10}}^{a} \operatorname{Rain} \wedge L_{\geq \frac{9}{10}}^{a}\left(U_{\leq \frac{1}{10}}^{b} \text { Rain }\right)
$$

We also introduce the notions of lower and upper probability of a (possibly infinite) set of agents by introducing the operators $L_{\geq s}^{G}$ and $U_{>s}^{G}$. In our approach we assume that the agents can share their sets of probabilities in order to obtain a larger set that is available to all the members of the group.

It is worth clarifying the additional expressive power of our first-order logic, which we denote by ILUPFO, and it's comparison with [26]. The paper [26] introduces a logic whose syntax allows only Boolean combinations of formulas in which lower and upper probability operators are applied to first order sentences. On the other hand, here we use the most general approach, allowing

\footnotetext{
1 The notation is motivated by the logic LUPP from [27], where $L U P$ stands for "lower and upper probability", while the second $P$ indicates that the logic is propositional. We add $I$ to denote iteration of upper and lower operators.
} 
arbitrary combination of probability operators, so we can express the statement like "according to the agent a, the lower probability of rain in all cities is at least $\frac{1}{3}$ " $\left(L_{\geq \frac{1}{3}}^{a} \forall x\right.$ Rain $\left.(x)\right)$, but also "There exists a city in which it will surely not rain:"

$$
(\exists x) U_{=0}^{a} \operatorname{Rain}(x) .
$$

The appropriate modal semantics for our logics consists of a specific class of Kripke models, in which every world is equipped with sets of probability measures (one set for each agent).

We propose sound and strongly complete axiomatizations of the logics. Interestingly, we use the same axiomatizations that we used in [26], and we show that they are also complete for the richer logics presented here. Of course, the instances of the axiom schemata are different, because the sets of formulas of ILUPP and ILUPFO are larger, due to nesting of lower and upper probability operators, and due to the presence of more agents. Also, the definition of the syntactical consequence (proof) $\vdash$ is different, due to the different interpretation of classical formulas. Since the sets of formulas and the classes of models are different, the proof techniques are modified. In order to achieve completeness, we use a Henkin-like construction, following some of our earlier developed methods $[4,22,25,26,29]$.

In addition, we show how to extend the proposed axiomatizations in order to properly capture the notions of lower and upper probability of an infinite set of agents.

The interesting situation that one axiomatic system is sound and complete for more than one class of models is not an exception. For example, modal system $K$ is also sound and complete with respect to the class of all irreflexive models [14].

We also prove that the satisfiability problem for ILUPP logic is decidable. We combine the method of filtration [14] and a reduction to linear programming. In the first part of the proof, we show that a formula $\alpha$ is satisfiable in a world $w$ of an ILUPP model if and only if it is satisfiable in a finite model, i.e., a model with a finite number of worlds, bounded by a number which is a function of the length of $\alpha$, and such that the sets of probability measures are finite in every world of the model. Note that, while in a standard modal framework this is enough to prove decidability, since for every natural number $k$ there are only finitely many modal models with $k$ worlds, this is not the case for our logic. Indeed, since our models involve sets of probability measures, for every finite set of $k$ worlds, there are uncountably many probability measures defined on them, and uncountably many models with $k$ worlds. However, in the second part of the proof we use a reduction to linear programming to solve the probabilistic satisfiability in a finite number of steps.

Finally, we show that the logics proposed in this paper generalize the logics for reasoning about sharp probabilities from [23,24]. Indeed, we use an additional axiom scheme to restrict the class of our models to the models isomorphic to those from[23,24]. We also formally infer all the axioms from [23, 24] using our extended axiomatization. 
The paper is organized as follows: in Section 2 we introduce the set of formulas of the logic ILUPP and we define the corresponding semantics. Then, in Section 3 we prove that the satisfiability problem for the logic ILUPP is decidable. In Section 4 we provide an axiomatic system ILUPP, and we prove that the axiomatization is strongly complete. The first-order logic ILUPFO is presented in section 5 . In section 6 , we show how to extend the presented axiomatic systems in case of infinite number of agents and we discuss decidability issues. We show that the proposed logics generalize the probability logics from $[23,24]$ in Section 7 . Finally, Section 8 contains some concluding remarks.

\section{The logic ILUPP}

In this section we introduce the syntax and the semantics of the logic ILUPP.

Let $\Sigma=\{a, b, \ldots\}$ be a finite, non-empty set of agents. Let $S=\mathbb{Q} \cap[0,1]$ and let $\mathcal{L}=\{p, q, r, \ldots\}$ be a denumerable set of propositional letters. The language of the logic ILUPP consists of the elements of set $\mathcal{L}$, propositional connectives $\neg$ and $\wedge$, and

- the list of upper probability operators $U_{\geq_{s}}^{a}$, for every $a \in \Sigma$ and $s \in S$,

- the list of lower probability operators $L_{\geq s}^{a}$, for every $a \in \Sigma$ and $s \in S$.

Definition 1 (Formula) The set For ILUPP of formulas is the smallest set containing all elements of $\mathcal{L}$ and that is closed under following formation rules: if $\alpha, \beta$ are formulas, then $L_{\geq s}^{a} \alpha, U_{\geq s}^{a} \alpha, \neg \alpha$ and $\alpha \wedge \beta$ are formulas as well. The formulas from For ILUPP will be denoted by $\alpha, \beta, \ldots$.

Intuitively, $U_{\geq s}^{a} \alpha$ means that according to an agent $a$, upper probability that a formula $\alpha$ is true is greater or equal to $s$ and analoguosly $L_{\geq s}^{a} \alpha$ means that according to an agent $a$ lower probability that a formula $\alpha$ is true is greater or equal to $s$.

Note that we use conjunction and negation as primitive connectives, while $\vee, \rightarrow$ and $\leftrightarrow$ are introduced in the usual way. We also use abbreviations to introduce other types of inequalities:

- $U_{<s}^{a} \alpha$ is $\neg U_{\geq s}^{a} \alpha, U_{\leq_{s}}^{a} \alpha$ is $L_{\geq 1-s}^{a} \neg \alpha, U_{=s}^{a} \alpha$ is $U_{\leq_{s}}^{a} \alpha \wedge U_{\geq_{s}}^{a} \alpha, U_{>s}^{a} \alpha$ is $\neg U_{\leq_{s}}^{a} \alpha$, - $L_{<s}^{a} \alpha$ is $\neg L_{\geq s}^{\bar{a}} \alpha, L_{\leq s}^{a} \alpha$ is $U_{\geq 1-s}^{\bar{a}} \neg \alpha, L_{=s}^{a} \alpha$ is $L_{\leq s}^{\bar{a}} \alpha \wedge L_{\geq s}^{\bar{a}_{s}} \alpha, L_{>s}^{a} \alpha$ is $\neg L_{\leq s}^{\bar{a}} \alpha$.

For example, the expression

$$
p \wedge U_{=0.9}^{a} L_{=0.3}^{b}(p \vee q)
$$

is a formula of our language.

Furthermore, we introduce the additional operators $L_{\geq s}^{G}$ and $U_{\geq s}^{G}$ that can speak about upper/lower probability of a group of agents $G$. We assume that the agents can share their sets of probabilities in order to obtain a larger set that is available to all the members of the group. Naturally, upper probability of an event w.r.t. that set will be at least upper probability of an individual agent; similarly, its lower probability is at most lower probability of a member of the group. Following that intuition, we introduce the abbreviations: 
- $L_{\geq_{s}}^{G} \alpha$ is the formula $\bigwedge_{a \in G} L_{\geq s}^{a} \alpha$,

- $U_{\geq s}^{\bar{G}} \alpha$ is the formula $\bigvee_{a \in G} U_{\geq s}^{\bar{a}} \alpha$.

We also introduce the corresponding operators with other types of inequalities, in the same way as we have done it for individual agents.

This formalization is in tune with the intuition behind the example given in the introduction, where the agent $a$ is reasoning in a skeptical way: she will visit a city as the probability of rain is at most $\frac{1}{10}$, according to all weather reports she considers. Then, if the agent $b$ shares a new, more negative report coming from a different source (e.g. probability of rain is $\frac{1}{5}$ ), a can revise her decision based on the updated upper probability.

At first glance, the asymmetry between $L_{\geq s}^{G}$ and $U_{\geq s}^{G}$ (conjunction vs disjunction) might look counter-intuitive. On the contrary, we believe that using the same type of connective (e.g. conjunction, which would be in the spirit of the epistemic operator $E_{G}$ "everyone in the group $G$ knows") would not fit the general intuition behind upper and lower probabilities: if increasing (resp. decreasing) upper probability comes from enlarging (narrowing) the set of probabilities, then lower probability should decrease (increase). ${ }^{2}$ Moreover, we will see that our definition of $L_{\geq s}^{G}$ and $U_{\geq s}^{G}$ implies that the groups semantically behave like individual agents.

In the last section, we discuss the alternative possibility for defining lower and upper probability of a group.

The semantics of the logic ILUPP is based on the possible-world approach. Every world is equipped with an evaluation function on propositional letters, and one generalized probability space for each agent.

Definition 2 (ILUPP-structure) An ILUPP-structure is a tuple $\langle W, L U P, v\rangle$, where:

- W is a nonempty set of worlds,

- LUP assigns, to every $w \in W$ and every $a \in \Sigma$, a space, such that

$\operatorname{LUP}(a, w)=\langle W(a, w), H(a, w), P(a, w)\rangle$, where:

$-\emptyset \neq W(a, w) \subseteq W$,

- H(a,w) is an algebra of subsets of $W(a, w)$, i.e. a set of subsets of $W(a, w)$ such that:

- $W(a, w) \in H(a, w)$,

- if $A, B \in H(a, w)$, then $W(a, w) \backslash A \in H(a, w)$ and $A \cup B \in H(a, w)$,

- $P(a, w)$ is a set of finitely additive probability measures defined on $H(a, w)$, i.e. for every $\mu(a, w) \in P(a, w), \mu(a, w): H(a, w) \longrightarrow[0,1]$ and the following conditions hold:

- $\mu(a, w)(W(a, w))=1$,

- $\mu(a, w)(A \cup B)=\mu(a, w)(A)+\mu(a, w)(B)$, whenever $A \cap B=\emptyset$.

2 Also, note that the notions conjunctive and disjunctive are relative in this specific context, since the type of connective is closely related to the type of inequality that an operator uses. For example, the definition $U_{\geq s}^{G} \alpha \equiv \bigvee_{a \in G} U_{\geq s}^{a} \alpha$ is equivalent to the "conjunctive" definition $U_{\leq s}^{G} \alpha \equiv \bigwedge_{a \in G} U_{\leq s}^{a} \alpha$. 
- $v: W \times \mathcal{L} \longrightarrow\{$ true, false $\}$ provides for each world $w \in W$ a two-valued evaluation of the primitive propositions.

Now we define satisfiability of the formulas from For ILUPP in the worlds of ILUPP-structures. As we mentioned in the introduction, for any set $P$ of probability measures defined on given algebra $H$, the lower probability measure $P_{\star}$ and the upper probability measure $P^{\star}$ are defined by

$-P_{\star}(X)=\inf \{\mu(X) \mid \mu \in P\}$ and

- $P^{\star}(X)=\sup \{\mu(X) \mid \mu \in P\}$,

for every $X \in H$. It is easy to check that

$$
P_{\star}(X)=1-P^{\star}\left(X^{c}\right),
$$

for every $X \in H$. In the context of the definition of an ILUPP-structure, we will denote $P_{\star}(a, w)\left([\alpha]_{M, w}^{a}\right)=\inf \left\{\mu\left([\alpha]_{M, w}^{a}\right) \mid \mu \in P(a, w)\right\}$ and $P^{\star}(a, w)\left([\alpha]_{M, w}^{a}\right)=$ $\sup \left\{\mu\left([\alpha]_{M, w}^{a}\right) \mid \mu \in P(a, w)\right\}$, where $[\alpha]_{M, w}^{a}=\{u \in W(a, w) \mid M, u \models \alpha\}$.

Definition 3 (Satisfiability relation) For every ILUPP structure $M=$ $\langle W, L U P, v\rangle$ and every $w \in W$, the satisfiability relation $\models$ fulfills the following conditions:

- if $p \in \mathcal{L}, M, w \models p$ iff $v(w)(p)=$ true,

$-M, w=\neg \alpha$ iff it is not the case that $M, w \models \alpha$,

- $M, w=\alpha \wedge \beta$ iff $M, w \models \alpha$ and $M, w \models \beta$,

- $M, w=U_{\geq s}^{a} \alpha$ iff $P^{\star}(a, w)\left([\alpha]_{M, w}^{a}\right) \geq s$,

- $M, w \mid=L_{\geq s}^{\bar{a}} \alpha$ iff $P_{\star}(a, w)\left([\alpha]_{M, w}^{a}\right) \geq s$.

We will omit $M$ when it's clear from context. Obviously, the operators indexed by groups satisfy the conditions:

- $M, w \mid=L_{\geq_{s}}^{G} \alpha$ iff $M, w \models L_{\geq_{s}}^{a} \alpha$ for all $a \in G$,

- $M, w \mid=U_{\geq s}^{G} \alpha$ iff $M, w \models U_{\geq s}^{a} \alpha$ for some $a \in G$.

Remark 1 Our logic has two types of basic operators for describing lower and upper probabilities. However, from the semantical point of view, lower probabilities can be inferred from upper probabilities, according to the equation (1). That fact will impact the presentation of the results in this paper. For example, in the proofs that use induction on the complexity of a formula to prove its semantical properties, the case when the formula is of the form $L_{\geq s}^{a} \alpha$ is an easy consequence of the case when we consider $U_{\geq s}^{a} \alpha$ (as an illustration, see the last paragraph of the proof of Theorem 2). Another consequence of the equation (1) is that the canonical model (Definition 1), used for the proof of completeness, can be defined using upper probabilities only.

It can be shown that satisfiability of the operators indexed by groups can be characterized by the set of probability measures that collect all the measures of individual members of the group. Indeed, if $P(G, w)$ denotes the set $\bigcup_{a \in G} P(a, w)$, we can observe that 
$-M, w \mid=U_{\geq s}^{G} \alpha$ iff $P^{\star}(G, w)\left([\alpha]_{M, w}^{a}\right) \geq s$,

$-M, w \mid=L_{\geq s}^{\bar{G}} \alpha$ iff $P_{\star}(G, w)\left([\alpha]_{M, w}^{a}\right) \geq s$.

It is clear from these two conditions that the behavior of the operators indexed by groups is similar to behavior of operators for individual agents. Indeed, they also represent upper and lower probability of a set of probabilities.

Note that this semantical interpretation of satisfiability does not use the fact that $G$ is finite, and it will also be applicable in Section 6 , where we will consider infinite groups of agents.

The possible problem with Definition 3 is that it might happen that for some $M, w, a$ and $\alpha$ the set $[\alpha]_{M, w}^{a}$ doesn't belong to $W(a, w)$. For that reason, we will consider only so called measurable structures.

Definition 4 (Measurable structure) The structure $M$ is measurable if for every $a \in \Sigma$ and every $w \in W, H(a, w)=\left\{[\alpha]_{w} \mid \alpha \in\right.$ For $\left._{\text {ILUPP }}\right\}$. The class of all measurable structures of the logic ILUPP will be denoted by ILUPP Meas. $_{\text {. }}$.

Next we define satisfiability of an ILUPP-formula.

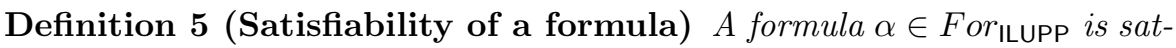

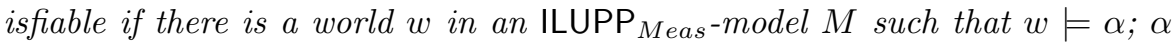
is valid if it is satisfied in every world in every ILUPP ${ }_{M e a s}$-model $M$. A set of formulas $T$ is satisfiable if there is a world $w$ in an ILUPP $_{M e a s}$-model $M$ such that $w \models \alpha$ for every $\alpha \in T$.

\section{Decidability of ILUPP}

In this section, we prove our main technical result. Recall the satisfiability problem: given an ILUPP-formula $\alpha$, we want to determine if there exists a

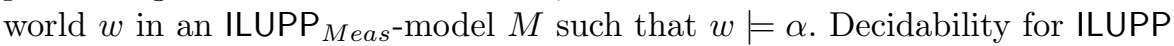
will be proved in two steps:

- first, we show that an ILUPP-formula is satisfiable iff it is satisfiable in a measurable structures with a finite number of worlds,

- second, we show that we can consider only finite measurable structures, i.e., measurable structure with finite number of worlds and with finite sets of probability measures in every world and for every agent, and

- third, we reduce the satisfiability problem in those finite models to a decidable linear programming problem.

In the first part of the proof, we will use the method of filtration [14]. Like the previous papers on the logical formalization of upper and lower probabilities $[11,26]$, we also use the characterization theorem by Anger and Lembcke [1]. It uses the notion of $(n, k)$-cover.

Definition $6((n, k)$-cover $) A$ set $A$ is said to be covered $n$ times by a multiset $\left\{\left\{A_{1}, \ldots, A_{m}\right\}\right\}$ of sets if every element of $A$ appears in at least $n$ sets from $A_{1}, \ldots, A_{m}$, i.e., for all $x \in A$, there exists $i_{1}, \ldots, i_{n}$ in $\{1, \ldots, m\}$ such that for all $j \leq n, x \in A_{i_{j}}$. An $(n, k)$-cover of $(A, W)$ is a multiset $\left\{\left\{A_{1}, \ldots, A_{m}\right\}\right\}$ that covers $W$ times and covers $A n+k$ times. 
Now we can state the characterization theorem.

Theorem 1 (Anger and Lembcke [1]) Let $W$ be a set, $H$ an algebra of subsets of $W$, and $f$ a function $f: H \longrightarrow[0,1]$. There exists a set $P$ of probability measures such that $f=P^{\star}$ iff $f$ satisfies the following three properties:

(1) $f(\emptyset)=0$,

(2) $f(W)=1$,

(3) for all natural numbers $m, n, k$ and elements $A_{1}, \ldots, A_{m}$ in $H$, if the multiset $\left\{\left\{A_{1}, \ldots, A_{m}\right\}\right\}$ is an $(n, k)$-cover of $(A, W)$, then $k+n f(A) \leq \sum_{i=1}^{m} f\left(A_{i}\right)$.

Let $S F(\alpha)$ denote the set of all subformulas of a formula $\alpha$, i.e.

$$
S F(\alpha)=\{\beta \mid \beta \text { is a subformula of } \alpha\} .
$$

Theorem 2 If a formula $\alpha$ is satisfiable, then it is satisfiable in an ILUPP Meas $^{-}$ model with at most $2^{|S F(\alpha)|}$ worlds.

Proof. Suppose that a formula $\alpha$ holds in some world of the model $M=$ $\langle W, L U P, v\rangle$ and let $k=|S F(\alpha)|$. By $\approx$, we will denote an equivalence relation over $W^{2}$, such that

$$
w \approx u \text { if and only if for every } \beta \in S F(\alpha), w \models \beta \text { iff } u \models \beta .
$$

Since there are finitely many subformulas of $\alpha$, we know that the quotient set

$$
W_{/ \approx}=\left\{C_{w_{i}} \mid w_{i} \in W\right\}
$$

is finite, where

$$
C_{w_{i}}=\left\{u \in W \mid u \approx w_{i}\right\}
$$

is the class of equivalence of $w_{i}$. More precsely,

$$
\left|W_{/ \approx}\right| \leq 2^{k}
$$

Next, from each class of equivalence $C_{w_{i}}$, we choose an element $w_{i}$.

Consider a tuple $\bar{M}=\langle\bar{W}, \overline{L U P}, \bar{v}\rangle$, where:

$-\bar{W}=\left\{w_{1}, w_{2}, \ldots\right\}$,

- For every $a$ and for every $w_{i} \overline{L U P}\left(a, w_{i}\right)=\left\langle\bar{W}\left(a, w_{i}\right), \bar{H}\left(a, w_{i}\right), \bar{P}\left(a, w_{i}\right)\right\rangle$ is defined as follows:

- $\bar{W}\left(a, w_{i}\right)=\left\{w_{j} \in \bar{W} \mid\left(\exists u \in C_{w_{j}}\right) u \in W\left(a, w_{i}\right)\right\}$

- $\bar{H}\left(a, w_{i}\right)=2^{\bar{W}\left(a, w_{i}\right)}$

- $\bar{P}\left(a, w_{i}\right)$ is any set of finitely additive measures, such that for every $D \in \bar{H}\left(a, w_{i}\right), \bar{P}^{\star}\left(a, w_{i}\right)(D)=P^{\star}\left(a, w_{i}\right)\left(\bigcup_{w_{j} \in D}\left(C_{w_{j}} \cap W\left(a, w_{i}\right)\right)\right)$

- $\bar{v}\left(w_{i}\right)(p)=v\left(w_{i}\right)(p)$, for every primitive proposition $p \in \mathcal{L}$.

First, we have to prove that $\bar{P}^{\star}\left(a, w_{i}\right)$ satisfies the conditions $(1)-(3)$ from Theorem 1, which will guarantee the existence of the sets $\bar{P}\left(a, w_{i}\right)$, for every agent $a$ and each $w_{i} \in \bar{W}$.

(1) $\bar{P}^{\star}\left(a, w_{i}\right)(\emptyset)=P^{\star}\left(a, w_{i}\right)\left(\bigcup_{w_{j} \in \emptyset}\left(C_{w_{j}} \cap W\left(a, w_{i}\right)\right)\right)=P^{\star}\left(a, w_{i}\right)(\emptyset)=0$; 
(2) $\bar{P}^{\star}\left(a, w_{i}\right)\left(\bar{W}\left(a, w_{i}\right)\right)=P^{\star}\left(a, w_{i}\right)\left(\bigcup_{w_{j} \in \bar{W}\left(a, w_{i}\right)}\left(C_{w_{j}} \cap W\left(a, w_{i}\right)\right)\right)=$ $=P^{\star}\left(a, w_{i}\right)\left(W\left(a, w_{i}\right)\right)=1$;

(3) Let $\left\{\left\{D_{1}, \ldots, D_{m}\right\}\right\}$ be an $(n, k)$-cover of $\left(D, \bar{W}\left(a, w_{i}\right)\right)$. That means:

i) every element from $D$ appears in at least $n+k$ sets from $D_{1}, \ldots, D_{m}$;

ii) every element from $\bar{W}\left(a, w_{i}\right)$ appears in at least $k$ sets from $D_{1}, \ldots, D_{m}$. Therefore,

iii) every element from $\left(\bigcup_{u \in D}\left(C_{u} \cap W\left(a, w_{i}\right)\right)\right.$ appears in at least $n+k$ sets from $\bigcup_{u \in D_{1}}\left(C_{u} \cap W\left(a, w_{i}\right)\right), \ldots, \bigcup_{u \in D_{m}}\left(C_{u} \cap W\left(a, w_{i}\right)\right)$;

iv) every element from $W\left(a, w_{i}\right)$ appears in at least $k$ sets from $\bigcup_{u \in D_{1}}\left(C_{u} \cap W\left(a, w_{i}\right)\right), \ldots, \bigcup_{u \in D_{m}}\left(C_{u} \cap W\left(a, w_{i}\right)\right)$.

Hence, by definition, we obtain that a multiset

$$
\left\{\left\{\bigcup_{u \in D_{1}}\left(C_{u} \cap W\left(a, w_{i}\right)\right), \ldots, \bigcup_{u \in D_{m}}\left(C_{u} \cap W\left(a, w_{i}\right)\right)\right\}\right\}
$$

is an $(n, k)$-cover of

$$
\left(\bigcup_{u \in D}\left(C_{u} \cap W\left(a, w_{i}\right)\right), W\left(a, w_{i}\right)\right) .
$$

Hence, using the fact that $P^{\star}\left(a, w_{i}\right)$ is an upper probability, from Theorem 1 , we have that

$$
k+n P^{\star}\left(a, w_{i}\right)\left(\bigcup_{u \in D}\left(C_{u} \cap W\left(a, w_{i}\right)\right)\right) \leq \sum_{j=1}^{m} P^{\star}\left(a, w_{i}\right)\left(\bigcup_{u \in D_{j}}\left(C_{u} \cap W\left(a, w_{i}\right)\right)\right),
$$

and therefore

$$
k+n \bar{P}^{\star}\left(a, w_{i}\right)(D) \leq \sum_{j=1}^{m} \bar{P}^{\star}\left(a, w_{i}\right)\left(D_{j}\right)
$$

Using induction on the complexity of a formula from the set $S F(\alpha)$, we can prove that for every $w \in \bar{W}$ and every $\beta \in S F(\alpha)$,

$$
M, w=\beta \quad \text { if and only if } \quad \bar{M}, w=\beta .
$$

If a formula is a propositional letter or obtained using Boolean connectives, the claim is trivial. So, let us consider the case when $\beta=U_{\geq s}^{a} \gamma$ :

$$
\begin{aligned}
M, w \models U_{\geq s}^{a} \gamma & \text { iff } \\
P^{\star}(a, w)(\{u \in W(a, w) \mid M, u \models \gamma\}) \geq s & \text { iff } \\
P^{\star}(a, w)\left(\bigcup_{M, u \models \gamma} C_{u} \cap W(a, w)\right) \geq s & \text { iff (ind. hyp.) } \\
\bar{P}^{\star}(a, w)\left(\left\{u \in \bar{W}^{\star}(a, w) \mid \bar{M}, u \models \gamma\right\}\right) \geq s & \text { iff } \\
\bar{M}, w \models U_{\geq s}^{a} \gamma . &
\end{aligned}
$$


At the end, let $\beta=L_{\geq s}^{a} \gamma$ :

$$
\begin{aligned}
& M, w \models L_{\geq s}^{a} \gamma \quad \text { iff } \\
& P_{\star}(a, w)(\{u \in W(a, w) \mid M, u \models \gamma\}) \geq s \quad \text { iff (equation (1)) } \\
& 1-P^{\star}(a, w)(\{u \in W(a, w) \mid M, u \models \neg \gamma\}) \geq s \quad \text { iff } \\
& 1-P^{\star}(a, w)\left(\bigcup_{M, u=\neg \gamma} C_{u} \cap W(a, w)\right) \geq s \quad \text { iff (ind. hyp.) } \\
& \bar{P}^{\star}(a, w)\left(\left\{u \in \bar{W}^{\star}(a, w) \mid \bar{M}, u \models \neg \gamma\right\}\right) \leq 1-s \quad \text { iff } \\
& \bar{M}, w \models U_{\leq 1-s}^{a} \neg \gamma \quad \text { iff } \\
& \bar{M}, w \models L_{\geq s}^{a} \gamma .
\end{aligned}
$$

In the second part of the proof, we use the following result of Halpern and Pucella [11].

Theorem 3 ([11]) Let $P$ be a set of probability measures defined on an algebra $H$ over a finite set $W$. Then there exists a set $P^{\prime}$ of probability measures such that, for each $X \in H, P^{*}(X)=\left(P^{\prime}\right)^{*}(X)$. Moreover, there is a probability measure $\mu_{X} \in P^{\prime}$ such that

$$
\mu_{X}(X)=P^{*}(X) .
$$

As a direct consequence of Theorem 2 and Theorem 3, we obtain the following result.

Lemma 1 If a formula $\alpha$ is satisfiable, then it is satisfiable in an ILUPP Meas $^{-}$ model with at most $2^{|S F(\alpha)|}$ worlds and for every agent $a \in \Sigma$ and every $w \in W, H(a, w)=2^{W(a, w)}$ and

$$
|P(a, w)|=|H(a, w)| .
$$

Furthermore, for each $X \in H(a, w)$, there exists a $\mu_{X} \in P(a, w)$ such that

$$
\mu_{X}(a, w)(X)=P^{*}(a, w)(X) .
$$

This lemma plays an important role in our proof of decidability. In the proof we will use the following notation: If $\alpha$ is an arbitrary formula, then

$$
S F(\alpha)=\left\{\beta_{1}, \ldots, \beta_{k}\right\} .
$$

In every $w \in W$, exactly one of the formulas of the following form:

$$
\pm \beta_{1} \wedge \cdots \wedge \pm \beta_{k}
$$

holds, where $\pm \beta_{i}$ denotes $\beta_{i}$ or $\neg \beta_{i}$. We will call that formula the characteristic formula for a world $w$. Also by $\beta \in\left(\alpha_{j}\right)^{+}$we will denote that $\beta$ is a conjunct in $\alpha_{j}$ and by $\beta \in\left(\alpha_{j}\right)^{-}$we will denote that $\neg \beta$ is a conjunct in $\alpha_{j}$. 
Theorem 4 Satisfiability problem for ILUPP Meas is decidable.

Proof. Let $M=\langle W, L U P, v\rangle$ be an ILUPP Meas -model and $\alpha$ an arbitrary formula. If $k$ is the cardinality of the set $S F(\alpha)$, by Lemma 1 we know that there exists an ILUPP Meas -model $\bar{M}$ with

1) at most $2^{k}$ worlds and

2) at most $2^{2^{k}}$ probabilistic measures (for any agent and any world) such that for each measurable set $X$, there exists a probabilistic measure $\mu_{X}$ with

$$
\mu_{X}(a, w)(X)=P^{*}(a, w)(X),
$$

such that $\alpha$ holds in some world of the model $\bar{M}$ iff $\alpha$ holds in some world of a model $M$. Let us denote by ILUPP Meas $(k)$ the set of all measurable models which satisfy the conditions 1) and 2). Clearly, in order to check if $\alpha$ is satisfiable, it is sufficient to check if $\alpha$ is satisfied in a model from $\operatorname{ILUPP}_{\text {Meas }}(k)$. For every $l \leq 2^{k}$, we will consider models with

$-l$ worlds, $w_{1}, \ldots, w_{l}$, and

- for every agent $a$ and every world $w$, sets of probability measures $P(a, w)$, such that $|P(a, w)|=2^{|W(a, w)|}$, for every $W(a, w) \subseteq\left\{w_{1}, \ldots w_{l}\right\}$.

Recall that in each of these worlds, exactly one characteristic formula holds. Thus, without loss of generality, in this proof we identify worlds with their corresponding characteristic formulas. We will denote by $\alpha_{i}$ the characteristic formula for a world $w_{i}$.

Note that it is not the case that any formula of the form (2) is a characteristic formula of some world, since it might be propositionally inconsistent. We can formally check if the formula $\alpha_{i}$ of the form (2) is propositionally consistent using the following test:

(a) In $\alpha_{i}$ we replace every occurrence of a formula starting with a probabilistic operator with an atomic proposition (all the occurrences of the same formula are assigned the same atomic proposition). Then we obtain a propositional formula, $\alpha_{i}^{\prime}$. Using any algorithm for propositional satisfiability we check whether $\alpha_{i}^{\prime}$ is satisfiable. If $\alpha_{i}^{\prime}$ passes the test, then $\alpha_{i}$ is consistent.

We check if $\alpha$ is satisfied in a model from $\operatorname{ILUPP}_{\text {Meas }}(k)$ using the following procedure:

1) The procedure sets:

- The number of worlds $l$ such that $1 \leq l \leq 2^{k}$ (i.e., $W=\left\{w_{1}, \ldots, w_{l}\right\}$ );

$-l$ formulas $\alpha_{1}, \ldots, \alpha_{l}$ (not necessarily different) of the form

$$
\pm \beta_{1} \wedge \cdots \wedge \pm \beta_{k}
$$

where $S F(\alpha)=\left\{\beta_{1}, \ldots, \beta_{k}\right\}$ such each formula passes the consistency test (a), and such that $\alpha$ is a conjunct of at least one $\alpha_{i}$;

- an arbitrary subset of worlds $W\left(a, w_{i}\right) \subseteq\left\{w_{1}, \ldots w_{l}\right\}$, for every agent $a$ and every $w_{i} \in W$; 
2) Using the test described below, the procedure checks if there is a model $\bar{M}=\langle W, L U P, v\rangle$ of $\alpha$ from ILUPP $\operatorname{Ieas}_{\text {mas }}(k)$ with the set of worlds $W=$ $\left\{w_{1}, \ldots, w_{l}\right\}$ and their corresponding characteristic formulas $\alpha_{1}, \ldots, \alpha_{l}$, and such that $L U P$ contains all $W\left(a, w_{i}\right)$ 's

3 ) If the test succeeds, the formula $\alpha$ is satisfiable, and the procedure terminates, otherwise procedure sets different values at step 1) and repeats the test.

4) If the test fails for all the possibilities for $l, \alpha_{1}, \ldots, \alpha_{l}$ and $W\left(a, w_{i}\right)$ 's (for every $a$ and $w_{i}$ ), the formula $\alpha$ is not satisfiable.

Now it remains to describe the test (from step 2) which, for given

- number of worlds $l\left(1 \leq l \leq 2^{k}\right)$,

- characteristic formulas $\alpha_{1}, \ldots, \alpha_{l}$ of $l$ worlds, and

- sets of worlds $W\left(a, w_{i}\right) \subseteq\left\{w_{1}, \ldots w_{l}\right\}$

checks if there is a model of $\alpha$ from $\operatorname{ILUPP}_{\text {Meas }}(k)$, where $W=\left\{w_{1}, \ldots w_{l}\right\}$, with those characteristic formulas of worlds and those $W\left(a, w_{i}\right)$ 's in $L U P$. In the test we do not determine probability values precisely; we simply check if there are probability measures such that the probabilistic constraints are satisfied in corresponding worlds.

The test translates the problem to the problem of satisfiability of a set of linear equations and inequalities. Since the models from $\operatorname{ILUPP}_{\text {Meas }}(k)$ have finite sets of worlds, all the subsets of $W\left(a, w_{i}\right)$ will be measurable. Also, note that every $\mu\left(a, w_{i}\right) \in P\left(a, w_{i}\right)$ is of the form $\mu_{X}$ (recall that $\mu_{X}$ is such that $\left.\mu_{X}(a, w)(X)=P^{*}(a, w)(X)\right)$. The test considers the following set of linear equations and inequalities:

1) $y_{w_{i}, w_{j}}^{X, a} \geq 0$, for each $\mu_{X}\left(a, w_{i}\right) \in P\left(a, w_{i}\right)$ (i.e., for every $\left.X \subseteq W\left(a, w_{i}\right)\right)$ and $j=1, \ldots, l$;

2) $\sum_{w_{j} \in W\left(a, w_{i}\right)} y_{w_{i}, w_{j}}^{X, a}=1$, for every $\mu\left(a, w_{i}\right) \in P\left(a, w_{i}\right)$ (i.e., for every $\left.X \subseteq W\left(a, w_{i}\right)\right)$

3) $\sum_{w_{j} \in X} y_{w_{i}, w_{j}}^{X, a} \geq \sum_{w_{j} \in X} y_{w_{i}, w_{j}}^{Y, a}$, for every $X, Y \subseteq W\left(a, w_{i}\right)$;

4) $\sum_{w_{j}: \beta \in\left(\alpha_{j}\right)^{+}} y_{w_{i}, w_{j}}^{X, a} \geq s$, if $U_{\geq s}^{a} \beta \in \alpha_{i}, \quad X=\left\{w_{j} \mid \beta \in\left(\alpha_{j}\right)^{+}\right\}$;

5) $\sum_{w_{j}: \beta \in\left(\alpha_{j}\right)^{+}} y_{w_{i}, w_{j}}^{X, a}<s$, if $\neg U_{\geq s}^{a} \beta \in \alpha_{i}, \quad X=\left\{w_{j} \mid \beta \in\left(\alpha_{j}\right)^{+}\right\}$;

6) $\sum_{w_{j}: \beta \in\left(\alpha_{j}\right)^{-}} y_{w_{i}, w_{j}}^{X, a} \leq 1-s$, if $L_{\geq s}^{a} \beta \in \alpha_{i}, \quad X=\left\{w_{j} \mid \beta \in\left(\alpha_{j}\right)^{-}\right\}$;

7) $\sum_{w_{j}: \beta \in\left(\alpha_{j}\right)^{-}}^{w_{j}: \beta \in\left(\alpha_{j}\right)^{-}} y_{w_{i}, w_{j}}^{X, a}>1-s$, if $\neg L_{\geq s}^{a} \beta \in \alpha_{i}, X=\left\{w_{j} \mid \beta \in\left(\alpha_{j}\right)^{-}\right\}$,

where $y_{w_{i}, w_{j}}^{X, a}$ represents $\mu_{X}\left(a, w_{i}\right)\left(\left\{w_{j}\right\}\right)$.

- The first inequality states that all the measures must be nonnegative.

- The second equality assures that the probability of the set of all possible worlds has to be equal to 1 . 
- The third inequality corresponds to the fact that $\mu_{X}(a, w)(X)=P^{*}(a, w)(X)$ and therefore

$$
\mu_{X}(a, w)(X) \geq \mu(a, w)(X), \text { for all } \mu(a, w) \in P(a, w) .
$$

- For the fourth and fifth inequality, note that if $X=\left\{w_{j} \mid \beta \in\left(\alpha_{j}\right)^{+}\right\}$

$$
\sum_{w_{j}: \beta \in\left(\alpha_{j}\right)^{+}} \mu_{X}\left(a, w_{i}\right)\left(\left\{w_{j}\right\}\right)=P^{*}\left(a, w_{i}\right)\left([\beta]_{w_{i}}^{a}\right),
$$

so these inequalities reflect the appropriate constraints.

- In order to understand sixth and seventh inequality, first recall the equality connecting upper and lower probabilty:

$$
P^{*}\left([\neg \beta]_{w_{i}}^{a}\right)=1-P_{*}\left([\beta]_{w_{i}}^{a}\right) .
$$

Next, note that if $X=\left\{w_{j} \mid \beta \in\left(\alpha_{j}\right)^{-}\right\}$

$$
\sum_{w_{j}: \beta \in\left(\alpha_{j}\right)^{-}} \mu_{X}\left(a, w_{i}\right)\left(\left\{w_{j}\right\}\right)=P^{*}\left(a, w_{i}\right)\left([\neg \beta]_{w_{i}}^{a}\right) .
$$

Consequently, if

$$
P_{*}\left([\beta]_{w_{i}}^{a}\right) \geq s,
$$

then

$$
P^{*}\left([\neg \beta]_{w_{i}}^{a}\right) \leq 1-s,
$$

and similarly for the case when $P_{*}\left([\beta]_{w_{i}}^{a}\right)<s$.

The equations and inequalities 1-7 form a finite system of linear equalities and inequalities and it is well known that solving this system is decidable. If this system has a solution, then there exists a probabilistic space in each world and for every agent. Indeed, it can be defined in the following way:

$$
\mu_{X}\left(a, w_{i}\right)(S)=\sum_{w_{j} \in S} y_{w_{i}, w_{j}}^{X, a}
$$

for every $S \subseteq W\left(a, w_{i}\right)$. The opposite also holds: if the system doesn't have solutions, then the probabilistic spaces (in each world and for every agent) cannot exist. Indeed, otherwise we could use the probability measures $\mu_{X}\left(a, w_{i}\right)$ to define the solution of the system in the following way: $y_{w_{i}, w_{j}}^{X, a}=\mu_{X}\left(a, w_{i}\right)\left(\left\{w_{j}\right\}\right)$.

Also note that because of the condition $(a)$ above, we know that a valuation can be defined. Namely, there are no classical propositional contradictions in characteristic formulas and therefore there exists a valuation that gives adequate truth values to the propositional letters that appear in formulas and for all the other variables we can set arbitrary truth value, e.g. $\perp$. Moreover, in every world $w$ of the model, the characteristic formula of the world holds in $w$. Since $\alpha$ is a conjunct of at least one of the corresponding characteristic formulas, we have that $\alpha$ is satisfiable.

Note that in the previously described method we consider only finitely many systems of linear equation and inequalities. Therefore, the satisfiability 
problem is decidable.

Furthermore, $\alpha$ is valid if and only if $\neg \alpha$ is not ILUPP Meas -satisfiable, so we have also that problem of a validity of a formula is decidable as well.

\section{A complete axiomatization of ILUPP}

Having settled the decidability issue for the logic ILUPP, we turn to the problem of developing an axiomatic system for the logic ILUPP. That system will be denoted by $A x_{\text {ILUPP. }}$

We start with the observation that, like any other real-valued probabilistic logic, ILUPP is not compact. Indeed, consider the set of formulas $T=$ $\left\{\neg U_{=0} \alpha\right\} \cup\left\{U_{<\frac{1}{n}} \alpha \mid n\right.$ is a positive integer $\}$. Obviously, every finite subset of $T$ is ILUPP ${ }_{\text {Meas }}$-satisfiable, but the set $T$ is not. Consequently, any finitary axiomatic system would be incomplete [32]. In order to achieve completeness, we use two infinitary rules of inference, with countably many premises and one conclusion.

In order to axiomatize upper and lower probabilities, we need to completely characterize them with a small number of properties. There are many complete characterizations in the literature, and the earliest appears to be by Lorentz [19]. We will use Theorem 1 from the previous section.

For the logic ILUPP, we use the axiomatic system for the logic LUPP from [26]. We only need to modify the form of the axioms due to presence of multiple agents. Apart from that, it should be also mentioned that the instances of axioms are different, since in [26] the operators of upper and lower probability are applied to classical formulas only, while here their nesting is allowed.

Axiom schemes

(A1) all instances of the classical propositional tautologies

(A2) $U_{<1}^{a} \alpha \wedge L_{<1}^{a} \alpha$

(A3) $U_{<r}^{<} \alpha \rightarrow \bar{U}_{<s}^{a} \alpha, s>r$

(A4) $U_{<s}^{\vec{a}} \alpha \rightarrow U_{\leq s}^{a} \alpha$

(A5) $\left(U_{\leq r_{1}}^{a} \alpha_{1} \wedge \cdots \wedge U_{\leq r_{m}}^{a} \alpha_{m}\right) \rightarrow U_{\leq r}^{a} \alpha$, if $\alpha \rightarrow \bigvee_{J \subseteq\{1, \ldots, m\},|J|=k+n} \bigwedge_{j \in J} \alpha_{j}$ and $\bigvee_{J \subseteq\{1, \ldots, m\},|J|=k} \bigwedge_{j \in J} \alpha_{j}$ are instances of the classical propositional tautologies, where $r=\frac{\sum_{i=1}^{m} r_{i}-k}{n}, n \neq 0$

(A6) $\neg\left(U_{\leq r_{1}}^{a} \alpha_{1} \wedge \cdots \wedge U_{\leq r_{m}}^{a} \alpha_{m}\right)$, if $\bigvee_{J \subseteq\{1, \ldots, m\},|J|=k} \bigwedge_{j \in J} \alpha_{j}$ is an instance of the classical propositional tautology and $\sum_{i=1}^{m} r_{i}<k$

(A7) $L_{=1}^{a}(\alpha \rightarrow \beta) \rightarrow\left(U_{\geq s}^{a} \alpha \rightarrow U_{\geq s}^{a} \beta\right)$

Inference Rules

(1) From $\alpha$ and $\alpha \rightarrow \beta$ infer $\beta$

(2) From $\alpha$ infer $L_{\geq 1}^{a} \alpha$ 
(3) From the set of premises

$$
\left\{\alpha \rightarrow U_{\geq s-\frac{1}{k}}^{a} \beta \mid k \in \mathbb{N}, k \geq \frac{1}{s}\right\}
$$

infer $\alpha \rightarrow U_{\geq s}^{a} \beta$

(4) From the set of premises

$$
\left\{\alpha \rightarrow L_{\geq s-\frac{1}{k}}^{a} \beta \mid k \in \mathbb{N}, k \geq \frac{1}{s}\right\}
$$

infer $\alpha \rightarrow L_{\geq s}^{a} \beta$.

The axioms $\mathrm{A} 5$ and $\mathrm{A} 6$ together capture the third condition from the Theorem 1. Indeed, as explained in [26], " $\left\{\left\{A_{1}, \ldots, A_{m}\right\}\right\}$ covers a set $A n$ times" can be formally written as $A \subseteq \bigcup_{J \subseteq\{1, \ldots, m\},|J|=n} \bigcap_{j \in J} A_{j}$. Thus, the condition that $\alpha \rightarrow \bigvee_{J \subseteq\{1, \ldots, m\},|J|=k+n} \bigwedge_{j \in J} \alpha_{j}$ is an instance of a propositional tautology ensures that $[\alpha]$ is covered $n+k$ times by a multiset $\left\{\left\{\left[\alpha_{1}\right], \ldots,\left[\alpha_{m}\right]\right\}\right\}$, while the condition that $\bigvee_{J \subseteq\{1, \ldots, m\}, J \mid=k} \bigwedge_{j \in J} \alpha_{j}$ is a propositional tautology gives us that $W=[\top]$ is covered $k$ times by a multiset $\left\{\left\{\left[\alpha_{1}\right], \ldots,\left[\alpha_{m}\right]\right\}\right.$.

The rules (3) and (4) are infinitary rules of inference and intuitively state that if an upper/lower probability is arbitrary close to a rational number $s$ then it is at least $s$.

Now we define some proof theoretical notions.

- $\vdash \alpha$ ( $\alpha$ is a theorem) iff there is an at most denumerable sequence of formulas $\alpha_{1}, \alpha_{2}, \ldots, \alpha$, such that every $\alpha_{i}$ is an axiom or it is derived from the preceding formulas by an inference rule;

- $T \vdash \alpha$ ( $\alpha$ is derivable from $\mathrm{T}$ ) if there is an at most denumerable sequence of formulas $\alpha_{1}, \alpha_{2}, \ldots, \alpha$, such that every $\alpha_{i}$ is an axiom or a formula from the set $T$, or it is derived from the preceding formulas by an inference rule, with the exception that Inference Rule 2 can be applied only to the theorems;

- $T$ is consistent if there is at least one formula $\alpha \in$ For ILUPP that is not deducible from $T$, otherwise $T$ is inconsistent;

- $T$ is maximal consistent set if it is consistent and for every $\alpha \in$ For ILUPP, $_{\text {, }}$ either $\alpha \in T$ or $\neg \alpha \in T$;

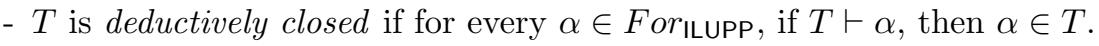

Note that $T$ is inconsistent iff $T \vdash \perp$. Also, it is easy to check that every maximal consistent set is deductively closed.

Due to similarity between this axiomatic system and the axiomatization from [26], many parts of the proof of completeness theorem are similar to the proofs that we already presented in [26], but there are also several differences, since the sets of formulas are not the same and the models are different. In the rest of this section, we present novel proofs and also reuse some ideas from [26] for readability, while we omit some parts that are identical to the proofs from $[26]$. 


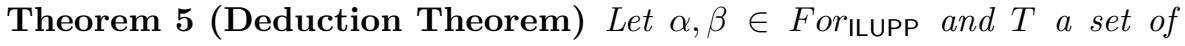
formulae. Then $T, \alpha \vdash \beta$ implies $T \vdash \alpha \rightarrow \beta$.

Proof. By transfinite induction on the length of the proof of $\beta$. The cases when $\vdash \beta, \beta=\alpha$ or $\beta$ is obtained by modus ponens are standard. So, let $\beta=L_{>1}^{a} \gamma$ be obtained from $T \cup\{\alpha\}$ by an application of Rule (2). Since the application of the inference Rule (2) is restricted to theorems only, we have:

(1) $\vdash \gamma$

(2) $T \vdash \gamma$

(3) $T \vdash L_{\geq 1}^{a} \gamma \quad$ by Rule (2)

(4) $T \vdash L_{\geq 1}^{a} \gamma \rightarrow\left(\alpha \rightarrow L_{\geq 1}^{a} \gamma\right)$ propositional reasoning

(5) $T \vdash \alpha \rightarrow L_{\geq 1}^{a} \gamma \quad$ by Rule (1).

Now, let us consider the case where $\beta=\beta_{1} \rightarrow L_{\geq s}^{a} \gamma$ is obtained from $T \cup\{\alpha\}$ by an application of Rule (4). Then:

(1) $T, \alpha \vdash \beta_{1} \rightarrow L_{\geq s-\frac{1}{k}}^{a} \gamma$, for all $k \geq \frac{1}{s}$

(2) $T \vdash \alpha \rightarrow\left(\beta_{1} \rightarrow L_{\geq s-\frac{1}{k}}^{a} \gamma\right)$, by the induction hypothesis

(3) $T \vdash\left(\alpha \wedge \beta_{1}\right) \rightarrow L_{\geq s-\frac{1}{k}}^{a} \gamma$, propositional reasoning

(4) $T \vdash\left(\alpha \wedge \beta_{1}\right) \rightarrow L_{\geq s}^{a} \gamma$, by Rule (4)

(5) $T \vdash \alpha \rightarrow \beta$, propositional reasoning.

Finally, the case where $\beta=\beta_{1} \rightarrow U_{\geq s}^{a} \gamma$ is obtained from $T \cup\{\alpha\}$ by an application of Rule (3) is analogous.

It is easy to check that the axiomatic system $A x_{\text {ILUPP }}$ is sound with respect to the class of ILUPP Meas -models.

We prove that the axiomatization $A x_{\text {ILUPP }}$ is complete, using a Henkin-like construction. Due to the presence of infinitary rules, the standard completion technique (Lindenbaum's theorem) has to be modified in the following way: if the current theory is inconsistent with the current formula and that formula can be derived by one of infinitary inference rules, than one of the premises must be blocked (see the proof of Theorem 6 ).

Definition 1 (Canonical Model) Canonical model $M_{C a n}=\langle W, L U P, v\rangle$ where:

- $W=\{w \mid w$ is a maximal consistent set of formulas $\}$,

- for every world $w$ and every propositional letter $p, v(w)(p)=$ true iff $p \in w$,

- for every $a \in \Sigma$ and $w \in W, L U P(a, w)=\langle W(a, w), H(a, w), P(a, w)\rangle$ is defined in the following way:

- $W(a, w)=W$,

- H(a,w) $=\left\{\{u \mid u \in W(a, w), \alpha \in u\} \mid \alpha \in\right.$ For $\left._{\mathrm{ILUPP}}\right\}$,

- $P(a, w)$ is any set of probability measures such that $P^{\star}(a, w)(\{u \mid u \in W(a, w), \alpha \in u\})=\sup \left\{s \mid U_{\geq s} \alpha \in w\right\}$

Lemma 2 For every $a \in \Sigma$, every $w \in W$ and every formula $\alpha$,

$$
\{u \mid u \in W(a, w), \alpha \in u\}=[\alpha]_{w}^{a} .
$$


Proof. We prove the statement by proving that $\alpha \in u$ iff $u \models \alpha$ by induction on the length of $\alpha$. If $\alpha=p$ the claim follows by definition of the canonical model. Cases when $\alpha=\neg \beta$ or $\alpha=\beta \wedge \gamma$ are trivial. Let us consider the case when $\alpha=U_{\geq s}^{a} \beta$. If $\alpha \in u$ then

$$
\sup \left\{r \mid U_{\geq r}^{a} \beta \in u\right\}=P^{\star}(a, u)(\{v \mid v \in W(a, u), \beta \in v\}) \geq s,
$$

and so $u \models \alpha$. Now, suppose that $u \models U_{\geq s}^{a} \beta$, i.e.

$$
\sup \left\{r \mid U_{\geq r}^{a} \beta \in u\right\} \geq s .
$$

a) If $\sup \left\{r \mid U_{>r}^{a} \beta \in u\right\}>s$, then by the properties of supremum and monotonicity of $\bar{P}^{\star}(a, u)$ we obtain $U_{\geq s} \alpha \in u$.

b) If $\sup \left\{r \mid U_{>r}^{a} \beta \in u\right\}=s$, then, as a direct consequence of inference Rule 3 , we have that $U_{\geq s}^{a} \alpha \in u$.

The case when $\alpha=L_{\geq s}^{a} \beta$ can be proved using equation (1).

Lemma $3 M_{C a n}$ is a well defined measurable structure.

Proof. From the Lemma 2 and the fact that $P^{\star}(a, w)$ is an upper probability measure (the proof that $P^{\star}(a, w)$ is an upper probability measure follows from Theorem 1 and the axioms A5 and A6 and it is essentially the same as the proof of the Lemma 3 in [26]) we obtain that $M_{C a n}$ is a well defined measurable structure.

Theorem 6 (Lindenbaum's theorem) Every consistent set of formulas can be extended to a maximal consistent set.

We assume an enumeration $\alpha_{0}, \alpha_{1}, \ldots$ of all formulas and define the chain of sets $T_{i}, i=0,1,2, \ldots$ and the set $T^{\star}$ in the following way:

(1) $T_{0}=T$,

(2) for every $i \geq 0$,

(a) if $T_{i} \cup\left\{\alpha_{i}\right\}$ is consistent, then $T_{i+1}=T_{i} \cup\left\{\alpha_{i}\right\}$, otherwise

(b) if $\alpha_{i}$ is of the form $\beta \rightarrow U_{\geq s}^{a} \alpha$, then $T_{i+1}=T_{i} \cup\left\{\neg \alpha_{i}, \beta \rightarrow \neg U_{\geq s-\frac{1}{n}}^{a} \alpha\right\}$, for some positive integer $n$, so that $T_{i+1}$ is consistent, otherwise

(c) if $\alpha_{i}$ is of the form $\beta \rightarrow L_{\geq s}^{a} \alpha$, then $T_{i+1}=T_{i} \cup\left\{\neg \alpha_{i}, \beta \rightarrow \neg L_{\geq s-\frac{1}{n}}^{a} \alpha\right\}$, for some positive integer $n$, so that $T_{i+1}$ is consistent, otherwise

(d) $T_{i+1}=T_{i} \cup\left\{\neg \alpha_{i}\right\}$.

(3) $T^{\star}=\bigcup_{i=0}^{\infty} T_{i}$.

The proof that $T^{\star}$ is a maximal consistent set is based on the following observations:

i) Natural numbers $(n)$, from the steps $2(b)$ and 2(c) of the construction exist; this follows from the Theorem 5 .

ii) Each $T_{i}$ is consistent, by construction.

iii) $T^{\star}$ does not contain all the formulas, by construction, using the fact that all $T_{i}$ 's are consistent. 
$i v$ ) For every formula $\alpha$, either $\alpha \in T^{\star}$ or $\neg \alpha \in T^{\star}$, by construction (steps (1) and (2)).

$v$ ) For every formula $\alpha$, if $T^{\star} \vdash \alpha$, then $\alpha \in T^{\star}$. The proof of this fact is by the induction on the length of the inference. Suppose that the sequence $\gamma_{1}, \gamma_{2}, \ldots, \alpha$ is the proof of $\alpha$ from $T^{\star}$. We show only the case when the sequence is countably infinite. The idea is to prove that, for every $i$, if $\gamma_{i}$ is obtained by an application of an arbitrary inference rule, and all the premises belong to $T^{\star}$, then, also $\gamma_{i} \in T^{\star}$. Let us consider the infinitary Rule 4. Let $\gamma_{i}=\beta \rightarrow L_{\geq s}^{a} \alpha$ be obtained from the set of premises $\left\{\gamma_{i}^{k}=\beta \rightarrow\right.$ $\left.L_{\geq s_{k}}^{a} \alpha \mid s_{k}=s-\frac{1}{k}, k>\frac{1}{s}, k \in \mathbb{N}\right\}$. By the induction hypothesis, we have that $\gamma_{i}^{k} \in T^{\star}$, for every $k$. If $\gamma_{i} \notin T^{\star}$, by step (3)(b) of the construction, there are some $l$ and $j$ so that

$$
\neg\left(\beta \rightarrow L_{\geq s}^{a} \alpha\right), \beta \rightarrow \neg L_{\geq s-\frac{1}{l}}^{a} \alpha \in T_{j} .
$$

Thus, we have that for some $j^{\prime} \geq j$ :

$-\beta \wedge \neg L_{\geq s}^{a} \alpha \in T_{j^{\prime}}$,

$-\beta \in T_{j^{\prime}}$

$-\neg L_{\geq s-\frac{1}{l}}^{a} \alpha, L_{\geq s-\frac{1}{l}}^{a} \alpha \in T_{j^{\prime}}$.

Contradiction with the consistency of a set $T_{j^{\prime}}$. Similarly can be proved for the Inference Rule (3) and all the other cases are easier.

vi) Step $i v$ ) guarantees that $T^{\star}$ is maximal and from $v$ ) and $i i i$ ) we get that $T^{\star}$ is a deductively closed set that does not contain all the formulas, hence consistent.

Theorem 7 (Strong completeness) If $\alpha$ is a formula, and $T$ is a set of formulas of the logic ILUPP, then $T \vdash \alpha$ iff $T \models \alpha$.

Sketch of the proof. First we point out that the theorem follows from soundness of the axiomatic system $A x_{\text {ILUPP }}$, and the following usual formulation of strong completeness:

Every consistent set of formulas $T$ is satisfiable.

Recall that we extended $T$ to the maximal consistent set $T^{\star}$. We showed that for every formula $\alpha$, and every $w \in W, w \models \alpha$ iff $\alpha \in w$. Since $T^{\star} \in W$, we obtain $M_{C a n}, T^{\star} \models T$.

\section{The first-order logic ILUPFO}

In this section we briefly discuss the first-order case avoiding repetition of technical details mentioned for the propositional case.

The language of the logic ILUPFO consists of a denumerable set of variables $V a r=\{x, y, z, \ldots\}$, classical propositional connectives, universal quantifier $\forall$, for every integer $k \geq 0$, denumerably many function symbols $F_{0}^{k}, F_{1}^{k}, \ldots$ of arity $k$, denumerably many relation symbols $P_{0}^{k}, P_{1}^{k}, \ldots$ of arity $k$, the list of upper probability operators $U_{\geq s}^{a}$ and the list of lower probability operators 
$L_{\geq s}^{a}$. Functions of arity 0 will be called constants. Terms are defined as usual, as well as the notion of term that is free for a variable.

The set of formulas is the smallest set containing atomic formulas and that is closed under following formation rules: if $\alpha, \beta$ are formulas, then $L_{\geq s}^{a} \alpha$, $U_{\geq s}^{a} \alpha, \neg \alpha, \alpha \wedge \beta,(\forall x) \alpha$ are formulas as well.

An ILUPFO-structure is a tuple $\mathcal{M}=\langle W, D, I, L U P\rangle$, where:

- $W$ and $L U P$ are defined as in the propositional case,

- $D$ associates a non-empty domain $D(w)$ with every world $w \in W$,

- $I$ associates an interpretation $I(w)$ with every world $w \in W$ such that:

- $I(w)\left(F_{i}^{k}\right): D(w)^{k} \rightarrow D(w)$, for all $i$ and $k$,

- $I(w)\left(P_{i}^{k}\right) \subseteq D(w)^{k}$, for all $i$ and $k$.

Let $\mathcal{M}=\langle W, D, I, L U P\rangle$ be an ILUPFO-structure. A variable valuation $v$ assigns to every variable some element of the corresponding domain to every world $w \in W$, i.e. $v(w)(x) \in D(w)$. For $v, w \in W$ and $d \in D(w)$ we define $v_{w}[d / x]$ is a valuation same as $v$ except that $v_{w}[d / x](w)(x)=d$. The value of a term $t$, denoted by $I(w)(t)_{v}$ is defined as follows:

- if $t$ is a variable $x$, then $I(w)(x)_{v}=v(w)(x)$, and

- if $t=F_{i}^{m}\left(t_{1}, \ldots, t_{m}\right)$, then

$$
I(w)(t)_{v}=I(w)\left(F_{i}^{m}\right)\left(I(w)\left(t_{1}\right)_{v}, \ldots, I(w)\left(t_{m}\right)_{v}\right) .
$$

We consider a class of ILUPFO models that satisfy:

- all the worlds from a model have the same domain, i.e., for all $v, w \in W$, $D(v)=D(w)$,

- the terms are rigid, i.e., for every model their meanings are the same in all the worlds.

The truth value of a formula $\alpha$ in a world $w \in W$ of a model $\mathcal{M}=$ $\langle W, D, I, L U P\rangle$ for a given valuation $v$, denoted by $I(w)(\alpha)_{v}$ is defined as follows:

- if $\alpha=P_{i}^{m}\left(t_{1}, \ldots, t_{m}\right)$, then $I(w)(\alpha)_{v}=$ true if $\left\langle I(w)\left(t_{1}\right)_{v}, \ldots, I(w)\left(t_{m}\right)_{v}\right\rangle \in$ $I(w)\left(P_{i}^{m}\right)$, otherwise $I(w)(\alpha)_{v}=$ false,

- if $\alpha=\neg \beta$, then $I(w)(\alpha)_{v}=$ true if $I(w)(\beta)_{v}=$ false, otherwise $I(w)(\alpha)_{v}=$ false,

- if $\alpha=\beta \wedge \gamma$, then $I(w)(\alpha)_{v}=$ true if $I(w)(\beta)_{v}=$ true and $I(w)(\gamma)_{v}=$ true,

- if $\alpha=U_{\geq s}^{a} \beta$, then $I(w)(\alpha)_{v}=$ true if $P^{\star}(w, a)\left(\left\{u \in W(w, a) \mid I(u)(\beta)_{v}=\right.\right.$ true $\}) \geq s$, otherwise $I(w)(\alpha)_{v}=$ false,

- if $\alpha=L_{>_{s}}^{a} \beta$, then $I(w)(\alpha)_{v}=$ true if $P_{\star}(w, a)\left(\left\{u \in W(w, a) \mid I(u)(\beta)_{v}=\right.\right.$ true $\}) \geq s$, otherwise $I(w)(\alpha)_{v}=$ false,

- if $\alpha=(\forall x) \beta$, then $I(w)(\alpha)_{v}=$ true if for every $d \in D(w), I(w)(\beta)_{v_{w}[d / x]}=$ true, otherwise $I(w)(\alpha)_{v}=$ false.

A formula $\alpha$ holds in a world $w$ from a model $\mathcal{M}=\langle W, D, I, L U P\rangle$, denoted by $\mathcal{M}, w \models \alpha$, if for every valuation $v, I(w)(\alpha)_{v}=$ true. If $d \in D(w)$, we use $\mathcal{M}, w \models \alpha(d)$ to denote that $I(w)(\alpha(x))_{v_{w}[d / x]}=$ true, for every valuation $v$. 
A sentence $\alpha$ is satisfiable if there is a world $w$ in an ILUPFO-model $\mathcal{M}$ such that $\mathcal{M}, w \models \alpha$. A sentence $\alpha$ is valid if it is satisfied in every world in every ILUPFO-model $\mathcal{M}$. A set of sentences $T$ is satisfiable if there is a world $w$ in an ILUPFO-model $\mathcal{M}$ such that $\mathcal{M}, w=\alpha$ for every $\alpha \in T$.

We will use the notation ILUPFO Meas $_{\text {so }}$ to denote the class of all fixed domain measurable models with rigid terms, where by measurable model we mean that the set $\left\{u \in W(w, a) \mid I(u)(\alpha)_{v}=\right.$ true $\}$ of all worlds from $W(w, a)$ that satisfy $\alpha$ is measurable.

Axiomatic system for the logic ILUPFO contains all the axioms and inference rules from the section 4 plus the following axiom schemes:

(A8) $(\forall x)(\alpha \rightarrow \beta) \rightarrow(\alpha \rightarrow(\forall x) \beta)$, where the variable $x$ doesn't occur free in $\alpha$

(A9) $(\forall x) \alpha(x) \rightarrow \alpha(t)$, where $\alpha(t)$ is obtained by substitution of all free occurrences of $x$ in $\alpha(x)$ by the term $t$ which is free for $x$ in $\alpha(x)$

and the inference rule:

(5) From $\alpha$ infer $(\forall x) \alpha$.

In the completeness proof we can follow the ideas from the propositional case. Deduction theorem holds for $A x_{\text {ILUPFO }}$. Namely, the facts that our infinitary inference rules have implicative form, and that the application of Rule 3 is restricted to theorems only guarantee the proof. Also, we need a special kind of maximal consistent sets called saturated sets. $T$ is saturated if it is maximally consistent and satisfies the following condition:

$$
\text { if } \neg(\forall x) \alpha(x) \in T \text {, then for some term } t, \neg \alpha(t) \in T \text {. }
$$

Definition 7 (Canonical model) A canonical model $\mathcal{M}_{C a n}=\langle W, D, I, L U P\rangle$ is a tuple such that:

- W is the set of all saturated sets of formulas,

- $D$ is the set of all variable-free terms,

- for every $w \in W, I(w)$ is an interpretation such that:

- for every function symbol $F_{i}^{m}, I(w)\left(F_{i}^{m}\right): D^{m} \rightarrow D$ such that for all variable-free terms $t_{1}, \ldots, t_{m}, I(w)\left(F_{i}^{m}\right):\left\langle t_{1}, \ldots, t_{m}\right\rangle \mapsto F_{i}^{m}\left(t_{1}, \ldots, t_{m}\right)$,

- for every relation symbol $P_{i}^{m}, I(w)\left(P_{i}^{m}\right)=\left\{\left\langle t_{1}, \ldots, t_{m}\right\rangle \mid P_{i}^{m}\left(t_{1}, \ldots, t_{m}\right) \in\right.$ $w\}$, for all variable-free terms $t_{1}, \ldots, t_{m}$,

- for $a \in \Sigma$ and $w \in W, L U P(w, a)=\langle W(w, a), H(w, a), P(w, a)\rangle$ is defined:

- $W(w, a)=W$,

- $H(w, a)=\left\{\{u \mid u \in W(w, a), \alpha \in u\} \mid \alpha \in\right.$ For ILUPFO $_{\text {, }}$,

- $P(w, a)$ is any set of probability measures such that $P^{\star}(w, a)(\{u \mid u \in$ $W(w, a), \alpha \in u\})=\sup \left\{s \mid U_{\geq s}^{a} \alpha \in w\right\}$.

Analogously as in the propositional case it can be proved that the canonical model is indeed a model via: For every formula $\alpha$ and every $w \in W, \alpha \in w$ iff $w \models \alpha$. 
Theorem 8 (Lindenbaum's theorem) Every consistent set of formulas can be extended to a saturated set.

Sketch of the proof. Consider a consistent set $T$ and let $\alpha_{0}, \alpha_{1}, \ldots$ be an enu-

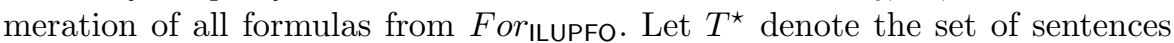
obtained by the steps (1)-(3) of the Theorem 6 with one additional requirement in the step (2):

if the set $T_{i+1}$ is obtained by adding a formula of the form $\neg(\forall x) \beta(x)$ to the set $T_{i}$, then for some $c \in C(C$ is a countably infinite set of new constant symbols), $\neg \beta(c)$ is also added to $T_{i+1}$, so that $T_{i+1}$ is consistent.

The new requirement produces consistent sets as well: suppose that for some $i>0$ the formula $\alpha_{i}$ is of the form $(\forall x) \beta(x)$ and that $T_{i} \cup\{(\forall x) \beta(x)\}$ is not consistent. Since $T_{i}$ is consistent, same holds for $T_{i} \cup\{\neg(\forall x) \beta(x)\}$. If there is a constant $c \in C$ such that $\neg \beta(c) \in T_{i}$, then obviously $T_{i} \cup\{(\forall x) \beta(x), \neg \beta(c)\}$ is consistent. Suppose that there is no such $c$. Since the set $T$ does not contain constants from $C$, and $T_{i} \cup\{\neg(\forall x) \beta(x)\}$ is obtained by adding only finitely many formulas to the set $T$, there must be at least one constant $c \in C$ such that $c$ does not appear in $T_{i} \cup\{\neg(\forall x) \beta(x)\}$. If $T_{i} \cup\{\neg(\forall x) \beta(x), \neg \beta(c)\}$ is not consistent, then $T_{i}, \neg(\forall x) \beta(x) \vdash \beta(c)$, and since $c$ does not appear in $T_{i} \cup\{\neg(\forall x) \beta(x)\}$, we obtain $T_{i}, \neg(\forall x) \beta(x) \vdash(\forall x) \beta(x)$. Thus, $T_{i} \vdash(\forall x) \beta(x)$. It follows that the set $T_{i}$ is not consistent, a contradiction.

At the end, we have to show that $T^{\star} \vdash \alpha$ implies $\alpha \in T^{\star}$, to prove that $T^{\star}$ is consistent, while the construction guarantees that $T^{\star}$ is both maximal and saturated. The only case that does not appear in the proof of the Theorem 6 concerns the situation when $T^{\star} \vdash(\forall x) \beta(x)$ is obtained from $T^{\star} \vdash \beta(x)$ by the inference Rule 5. Since $\beta(x)$ has one free variable, and $T_{i}$ and $T^{\star}$ are sets of sentences, $\beta(x)$ does not belong to $T^{\star}$. However, by classical reasoning, we have $T^{\star} \vdash \beta(c)$, for every constant $c \in C$, and from the induction hypothesis $\beta(c) \in T^{\star}$. If $(\forall x) \beta(x) \notin T^{\star}$, the construction of the set $T^{\star}$ guarantees that there has to be some $i>0$ such that $\beta(c), \neg \beta(c) \in T_{i}$ for some $c \in C$. Contradiction.

Theorem 9 (Strong completeness) Every consistent set of formulas $T$ is ILUPFO $_{\text {Meas }}$-satisfiable.

\section{Adding infinite number of agents}

In this section we extend the logics ILUPP and ILUPFO by considering a countable set of agents $\Sigma$. As previously pointed out by Halpern and Shore in [12], in many applications where the set of agents is not known in advance, and its cardinality has no a priori upper bound, it is easiest to model the set of agents as an infinite set.

In the rest of the section, we focus on extending ILUPP; nevertheless, combining this section with the first-order extension results from the previous section will straightforwardly lead to the extension of ILUPFO. 
In order to keep the language countable, we will consider a countable set $\mathcal{G}$ of subsets of $\Sigma$. We do not pose any constraint about the elements of $\mathcal{G}$ (like closeness under intersection etc.). We use the same operators $L_{\geq s}^{G}$ and $U_{\geq s}^{G}$ as before, now allowing that $G$ is any member of $\mathcal{G}$. Their semantical definition remains unchanged. However, the formulas of the form $L_{\geq s}^{G} \alpha$ and $U_{\geq s}^{G} \alpha$ now cannot be introduced as abbreviation, since we don't have infinite disjunctions and conjunctions in syntax. Therefore, we formally:

(a) extend the language with the operators $L_{\geq s}^{G}$ and $U_{\geq s}^{G}$, where $G \in \mathcal{G}$,

(b) extend the definition of satisfiability in order to capture the new operators:

$-M, w \models L_{\geq s}^{G} \alpha$ iff $M, w \models L_{\geq s}^{a} \alpha$ for all $a \in G$,

- $M, w \models U_{\geq s}^{\bar{G}} \alpha$ iff $M, w \models U_{\geq s}^{a} \alpha$ for some $a \in G$.

The extension of the logic ILUPP poses new axiomatization challenges. The fact that there is an infinite group of agents $G \in \mathcal{G}$ is an additional source of non-compactness of the logic. Indeed, the sets $\left\{L_{\geq_{s}}^{a} \beta \mid a \in G\right\} \cup\left\{L_{<s}^{G} \beta\right\}$ and $\left\{U_{<s}^{a} \beta \mid a \in G\right\} \cup\left\{U_{\geq s}^{G} \beta\right\}$ are finitely satisfiable, but unsatisfiable sets of formulas. For that reason, we will use additional inference rules, in order to ensure inconsistency of those sets and to obtain strong completeness.

Note that, once we allow infinite groups of agents, we cannot use the formulas $L_{\geq s}^{G} \alpha \leftrightarrow \bigwedge_{a \in G} L_{\geq s}^{a} \alpha$ and $U_{\geq s}^{G} \alpha \leftrightarrow \bigvee_{a \in G} U_{\geq s}^{a} \alpha$, as we did in Section 2, to capture semantical definitions above. Instead, we extend the axiomatization of ILUPP with the axioms

(A10) $L_{\geq s}^{G} \beta \rightarrow L_{\geq s}^{a} \beta$, if $a \in G$,

(A11) $U_{\geq s}^{\bar{a}} \beta \rightarrow U_{\geq s}^{\bar{G}} \beta$, if $a \in G$,

and the inference rules

(6) From the set of premises

$$
\left\{\alpha \rightarrow L_{\geq s}^{a} \beta \mid a \in G\right\}
$$

infer $\alpha \rightarrow L_{\geq s}^{G} \beta$

(7) From the set of premises

$$
\left\{\alpha \rightarrow U_{<s}^{a} \beta \mid a \in G\right\}
$$

infer $\alpha \rightarrow U_{<s}^{G} \beta$.

We will prove that this axiomatization is strongly complete. In the completeness proof we can follow the ideas from Section 4 . However, we need to extend the proofs of Theorem 5, Lemma 2 and Theorem 6.

In Theorem 5, we prove that $T, \alpha \vdash \beta$ implies $T \vdash \alpha \rightarrow \beta$, using the induction on the length of inference. Now we have two new inference rules. Here we consider the rule (6), while the proof for (7) is similar.

Assume that $T, \alpha \vdash \beta$ is obtained by the inference rule (6). Then $\beta$ is of the form $\alpha_{1} \rightarrow L_{>_{s}}^{G} \beta_{1}$, and we have $T, \alpha \vdash \alpha_{1} \rightarrow L_{\geq s}^{a} \beta_{1}$ for all $a \in G$. Consequently, $T \vdash\left(\alpha \wedge \alpha_{1}\right) \rightarrow L_{\geq s}^{a} \beta_{1}$ for all $a \in G$, by the induction hypothesis 
and simple propositional reasoning (i.e., $(p \rightarrow(q \rightarrow r)) \longleftrightarrow((p \wedge q) \rightarrow r))$. By the rule (6) we obtain $T \vdash\left(\alpha \wedge \alpha_{1}\right) \rightarrow L_{\geq_{s}}^{G} \beta_{1}$, or, equivalently, $T \vdash \alpha \rightarrow$ $\left(\alpha_{1} \rightarrow L_{\geq s}^{G} \beta_{1}\right)$. Thus, $T \vdash \alpha \rightarrow \beta$.

Next, in the proof of Lemma 2 we need to extend the proof that $\alpha \in u$ iff $u \models \alpha$. The proof was by induction on the complexity of $\alpha$. Since we now have a larger set of formulas, we need to consider the two additional cases in the proof: when $\alpha$ is of the form $L_{\geq s}^{G} \beta$ and when it is $U_{\geq s}^{G} \beta$. We assume that the operators of the group are of higher complexity then the operators of individual agents, for example $L_{\geq_{s}}^{G} \beta$ is more complex then $U_{\geq s}^{a} \beta$. It is sufficient to show that for every maximal consistent set $T^{\star}$ the following conditions hold:

(1) $L_{>s}^{G} \beta \in T^{\star}$ iff $\left\{L_{\geq s}^{a} \beta \mid a \in G\right\} \subseteq T^{\star}$,

(2) $U_{\geq s}^{\bar{G}} \beta \in T^{\star}$ iff $U_{\geq s}^{a} \beta \in T^{\star}$ for some $a \in G$.

Note that (1) follows directly from (A10) and the inference rule (6), and that $(\Leftarrow)$ part of $(2)$ follows from $($ A11). Let us prove $(\Rightarrow)$ part of $(2)$. Suppose that $U_{\geq_{s}}^{G} \beta \in T^{\star}$ and $U_{\geq_{s}}^{a} \beta \notin T^{\star}$ for every $a \in G$. By Maximality of $T^{\star}$, we have $\left\{U_{<s}^{a} \beta \mid a \in G\right\} \subseteq T^{\star}$. Then $T^{\star} \vdash U_{<s}^{G} \beta$ by the rule (7); a contradiction.

Finally, we need to modify the completion technique in the proof of Theorem 6 . Recall that the completion of $T$ is defined through an iterative process, assuming an enumeration $\alpha_{0}, \alpha_{1}, \ldots$ of all formulas. In the presence of the new rules, two new cases should be considered in the step (2), when $T_{i} \cup\left\{\alpha_{i}\right\}$ is inconsistent:

(e) if $\alpha_{i}$ is of the form $\alpha \rightarrow L_{>s}^{G} \beta$, then $T_{i+1}=T_{i} \cup\left\{\neg \alpha_{i}, \alpha \rightarrow L_{<s}^{a} \beta\right\}$, for some agent $a \in G$ such that $T_{i+1}$ is consistent,

(f) if $\alpha_{i}$ is of the form $\alpha \rightarrow U_{<s}^{G} \beta$, then $T_{i+1}=T_{i} \cup\left\{\neg \alpha_{i}, \alpha \rightarrow U_{\geq s}^{a} \beta\right\}$, for some agent $a \in G$ such that $T_{i+1}$ is consistent.

First, we need to show that each of the two conditions is correctly formulated, i.e., that there exists an agent $a \in G$ such that $T_{i+1}$ is consistent. This follows from Deduction theorem. For example, let us consider (e): if $T_{i+1}=T_{i} \cup\left\{\neg \alpha_{i}, \alpha \rightarrow L_{<s}^{a} \beta\right\}$ is inconsistent for all $a \in G$, then $T_{i} \cup\left\{\neg \alpha_{i}\right\} \vdash$ $\neg\left(\alpha \rightarrow L_{<s}^{a} \beta\right)$ for all $a \in G$. By (A1) we obtain $T_{i} \cup\left\{\neg \alpha_{i}\right\} \vdash \alpha \rightarrow L_{\geq s}^{a} \beta$ for all $a \in G$, therefore $T_{i} \cup\left\{\neg \alpha_{i}\right\} \vdash \alpha \rightarrow L_{\geq s}^{G} \beta$ by the inference rule (6).

Second, we need to prove that after adding the new inference rules, $T^{\star}$ is still deductively closed, i.e. $T^{\star} \vdash \alpha$ implies $\alpha \in T^{\star}$ (condition $v$ ) of the proof). Let us prove that $T^{\star}$ is closed under the rule (6). Let $\left\{\alpha \rightarrow L_{\geq s}^{a} \beta \mid\right.$ $a \in G\} \in T^{\star}$. Let $\alpha \rightarrow L_{\geq_{s}}^{G} \beta=\alpha_{i}$, and suppose that $\alpha \rightarrow L_{\geq_{s}}^{G} \beta \notin T^{\star}$. Then from the condition (e) we obtain $\neg\left(\alpha \rightarrow L_{x_{s}}^{G} \beta\right) \in T_{i+1}$; consequently, $T_{i+1} \vdash \alpha$. The same condition also ensures $\alpha \rightarrow L_{<s}^{\vec{a}} \beta \in T_{i+1}$, for some agent $a \in G$. Recall that $\alpha \rightarrow L_{\geq_{s}}^{a} \beta=\alpha_{j} \in T^{\star}$. Let $j$ be the positive integer such that $\alpha \rightarrow L_{\geq s}^{a} \beta=\alpha_{j}$. Then $T_{\max \{i+1, j\}} \vdash \alpha, T_{\max \{i+1, j\}} \vdash \alpha \rightarrow L_{\geq s}^{a} \beta$ and $T_{\max \{i+1, j\}} \vdash \alpha \rightarrow L_{<s}^{a} \beta$, so $T_{\max \{i+1, j\}} \vdash \perp$; a contradiction. The proof that $T^{\star}$ is closed under the rule (7) is similar.

At the end of this section, we discuss the decidability issue. We can prove that this logic is decidable, provided that we can check whether the sets that 
are obtained by finite set operations on elements of $\mathcal{G}$ are empty or not. The similar assumption is posed by Halpern and Shore in [12], where it is also observed that possibility of deciding the cardinality of the set $G \backslash\left(G_{1} \cup \cdots \cup G_{n}\right)$ in general depends on the way how $G, G_{1}, \ldots, G_{n}$ are presented and that if $G, G_{1}, \ldots, G_{n}$ are recursive sets, deciding if $G \backslash\left(G_{1} \cup \cdots \cup G_{n}\right)$ is empty may not even be recursive. If we assume that we have oracles for testing whether those sets are empty or not, we can, given a formula $\alpha$, detect all the nonempty sets obtained by applying set operations on groups mentioned in $\alpha .{ }^{3}$ Then we can replace all the agents that have same membership functions in those sets (i.e., that belong to exactly the same groups) by a single representative of the set. In that way, we modify $\alpha$ by replacing each infinite set of agents from $\alpha$ with a finite set. Then we can use finite conjunctions and disjunction to eliminate sets of agents, using the tautologies $L_{\geq s}^{G} \beta \leftrightarrow \bigwedge_{a \in G} L_{\geq s}^{a} \beta$ and $U_{\geq s}^{G} \beta \leftrightarrow \bigvee_{a \in G} U_{\geq s}^{a} \beta$. In that way we obtain a formula of the logic ILUPP and we can apply the procedure from Section 3 to check its satisfiability.

\section{ILUPP and ILUPFO as generalizations of probabilistic logics}

In this section we prove that the logics ILUPP and ILUPFO in a sense contain the logics LPP1 and LFOP1 (respectively) for reasoning about sharp probabilities [23,24]. The two logics presented here have the similar semantical structure as the logics LPP1 and LFOP1, since they both use Kripke-like structures. It is intuitively clear that the semantics of our logics are more general, since reasoning about upper and lower probabilities requires sets of probability measures, while in the logics for reasoning about sharp probabilities one measure per possible world is sufficient (and thus they are isomorphic to the "sets of" probability measures which are actually singletons). On the other hand, the axiomatic systems are quite different. Here we will focus on the two proof theoretical aspects of the generalization: first, which axioms should be added to our logics (both ILUPP and ILUPFO) to reduce the proposed class of models to the class of models isomorphic to the one for corresponding logic for sharp probabilities (LPP1 and LFOP1, respectively), and second, how we can use the added axioms to formally obtain the axiomatizations of LPP1 and LFOP1.

In the rest of the section we will focus on relation between the logics ILUPP and LPP1, but we stress that, similarly as in the previous section, analogous reasoning leads to the same relation in the first-order case, namely between the logics ILUPFO and LFOP1. First we state only those properties of the logic LPP1 important for this section (for more details about the logic LPP1, as well as for the logic LFOP1 we refer the reader to [23,24]).

The language of the logic LPP1 extends the classical propositional language with the list of operators $P_{\geq s}$, where $s$ is a rational number from the $[0,1]$. Concerning the syntax, besides the classical propositional formulas, formulas

\footnotetext{
3 Here, for simplicity, we can assume that all the singletons are in $\mathcal{G}$, in order to capture the operators indexed by individual agents.
} 
of the form $P_{\geq s} \alpha$ are also included. For example, $p \wedge P_{\leq \frac{1}{2}} q$ and $P_{=\frac{1}{3}} P_{\geq 1} p$ are well defined formulas. LPP1-structures are defined as triples $M=\langle W, \operatorname{Prob}, \nu\rangle$, where:

- $W$ is a non empty set of worlds

- Prob is a probability assignment which assigns to every $w \in W$ a probability space, such that $\operatorname{Prob}(w)=\langle W(w), H(w), \mu(w)\rangle$, where:

$W(w)$ is a non empty subset of $W$,

$H(w)$ is an algebra of subsets of $W(w)$ and

$\mu(w): H(w) \rightarrow[0,1]$ is a finitely additive probability measure.

$-\nu$ provides for each world $w$ an evaluation of the primitive propositions.

Satisfiability of a formula is defined as expected for the classical propositional formulas and

$$
M, w \models P_{\geq s} \alpha \text { iff } \mu(\{v \in W(w) \mid v \models \alpha\}) \geq s .
$$

Axiomatization of the logic LPP1 is the following:

(P1) all substitutional instances of the classical propositional tautologies,

(P2) $P_{\geq 0} \alpha$,

(P3) $P_{\leq r} \alpha \rightarrow P_{<s} \alpha, s>r$,

(P4) $P_{<s} \alpha \rightarrow P_{\leq s} \alpha$,

(P5) $\left(P_{\geq t} \alpha \wedge P_{\geq s} \beta \wedge P_{\geq 1}(\neg \alpha \vee \neg \beta)\right) \rightarrow P_{\geq \min \{1, t+s\}}(\alpha \vee \beta)$,

(P6) $\left(P_{\leq t} \alpha \wedge P_{<s} \beta\right) \rightarrow P_{<t+s}(\alpha \vee \beta), t+s \leq 1$.

Inference Rules

(1) Modus Ponens,

(2) from $\alpha$ infer $P_{\geq 1} \alpha$,

(3) from the set of premises

$$
\left\{A \rightarrow P_{\geq s-\frac{1}{k}} \alpha \mid k \geq \frac{1}{s}\right\}
$$

infer $A \rightarrow P_{\geq s} \alpha$.

Soundness and strong completeness theorems for the logic LPP1 are proved (for more details see [24], chapter 4).

We will now focus on the relationship between ILUPP and LPP1. First, since the logic LPP1 is not a multi-agent logic we put that the set of agents $\Sigma$ is a singleton set (so instead of $U_{\geq r}^{a} \alpha$ we will write $U_{\geq r} \alpha$ ).

Then, it is clear that the subclass of the ILUPP-structures that contains those structures where the set of finitely additive probability measures is a singleton set is isomorphic to the class of LPP1-structures. Therefore, we add the following axiom:

$$
\text { (A12) } \quad U_{\geq r} \alpha \rightarrow L_{\geq r} \alpha .
$$

We will denote ILUPP + Axiom $A 12$ by ILUPP ${ }^{E x t}$. 
Let us recall note that it is shown in [26] (Proposition 1) that the following formula is a theorem in our axiomatization:

$$
\vdash U_{\leq r} \alpha \rightarrow L_{\leq r} \alpha .
$$

From (3) and (4) follows that $U$ and $L$ have the same behavior in the sense that for every formula $\alpha$ and every $r \in S$

$$
\vdash U_{\geq r} \alpha \leftrightarrow L_{\geq r} \alpha .
$$

This means that in ILUPP ${ }^{E x t}$ one type of operators is sufficient, since changing one type of operator with other will lead to an equivalent formula. For example, if we replace all the operators for lower probability with the operators of upper probability in

we will obtain the formula

$$
\alpha \equiv L_{\geq \frac{1}{3}} U_{\leq \frac{1}{2}} L_{=1} p,
$$

$$
\beta \equiv U_{\geq \frac{1}{3}} U_{\leq \frac{1}{2}} U_{=1} p
$$

which is equivalent to $\alpha$. This holds for any formula and can be proved in a straightforward manner by the induction on the complexity of a formula. This fact allows us, without loss of generality, to consider only formulas with the $U$ operators in ILUPP ${ }^{E x t}$.

Our goal is to prove that the set of theorems of the logic LPP1 is a subset of the set of theorems of the logic ILUPP ${ }^{E x t}$. Therefore, we will prove that all the axioms and inference rules of the logic LPP1 can be inferred in the logic ILUPP $^{E x t}$, where $P$ is replaced by $U$.

Also notice that from the semantical point of view, addition of the axiom A12 guarantees that in an ILUPP-structure the set of finitely additive probability measures is a singleton set and therefore has the same form as the LPP1-structure.

The axioms (P1)-(P4) correspond to the axioms (A1)-(A4). It is also clear that the inference rules coincide as well. Our goal is to prove that the appropriate counterparts of the axioms (P5) and (P6), i.e.,

(U5) $\left(U_{\geq t} \gamma \wedge U_{\geq s} \beta \wedge U_{\geq 1}(\neg \gamma \vee \neg \beta)\right) \rightarrow U_{\geq \min \{1, t+s\}}(\gamma \vee \beta)$,

(U6) $\left(U_{\leq t} \gamma \wedge U_{<s} \beta\right) \rightarrow U_{<t+s}(\gamma \vee \beta), t+s \leq 1$,

follow from the axiomatization of ILUPP ${ }^{E x t}$, while in the inference the essential role play the axioms:

(A5) $\left(U_{\leq r_{1}}^{a} \alpha_{1} \wedge \cdots \wedge U_{\leq r_{m}}^{a} \alpha_{m}\right) \rightarrow U_{\leq r}^{a} \alpha$, if $\alpha \rightarrow \bigvee_{J \subseteq\{1, \ldots, m\},|J|=k+n} \bigwedge_{j \in J} \alpha_{j}$ and

$\bigvee_{J \subseteq\{1, \ldots, m\},|J|=k} \bigwedge_{j \in J} \alpha_{j}$ are tautologies, where $r=\frac{\sum_{i=1}^{m} r_{i}-k}{n}, n \neq 0$

(A6) $\neg\left(U_{\leq r_{1}}^{a} \alpha_{1} \wedge \cdots \wedge U_{\leq r_{m}}^{a} \alpha_{m}\right)$, if $\bigvee_{J \subseteq\{1, \ldots, m\},|J|=k} \bigwedge_{j \in J} \alpha_{j}$ is a tautology and $\sum_{i=1}^{m} r_{i}<k$.

In order to prove that we need the following Lemma:

Lemma 4 ILUPP $^{E x t} \vdash\left(U_{\leq t} \gamma \wedge U_{\leq s} \beta\right) \rightarrow U_{\leq t+s}(\gamma \vee \beta), t+s \leq 1$. 
Proof. We will show that for $t+s \leq 1$

$$
\left(U_{\leq t} \gamma \wedge U_{\leq s} \beta\right) \rightarrow U_{\leq t+s}(\gamma \vee \beta)
$$

can be infered from A5. Consider the axiom A5 for:

$$
\begin{aligned}
& m=2 ; n=1, k=0 ; r_{1}=t ; r_{2}=s ; \\
& \alpha_{1}=\gamma ; \quad \alpha_{2}=\beta ; \quad \alpha=\gamma \vee \beta .
\end{aligned}
$$

In this case we obtain $r=t+s$ and therefore the Axiom A5 has exactly the shape of the formula (6). We need also to check whether the formulas

$$
\alpha \rightarrow \bigvee_{J \subseteq\{1,2\},|J|=1} \bigwedge_{j \in J} \alpha_{j}
$$

and

$$
\bigvee_{J \subseteq\{1,2\},|J|=0} \bigwedge_{j \in J} \alpha_{j}
$$

are tautologies. The first formula has the form $\gamma \vee \beta \rightarrow \gamma \vee \beta$ which is clearly a tautology, while the second formula has the form $\bigwedge_{j \in \emptyset} \alpha_{j}$, and $\bigwedge_{j \in \emptyset} \alpha_{j}=\top$ by definition and hence a tautology.

Theorem 10 The set of theorems of the logic LPP1 is a subset of the set of theorems of the logic ILUPP ${ }^{E x t}$.

Proof. As already stated, we need only to prove that:

(a) ILUPP $^{E x t} \vdash\left(U_{\geq t} \gamma \wedge U_{\geq s} \beta \wedge U_{\geq 1}(\neg \gamma \vee \neg \beta)\right) \rightarrow U_{\geq \min \{1, t+s\}}(\gamma \vee \beta)$,

(b) $\operatorname{ILUPP}^{E x t} \vdash\left(U_{\leq t} \gamma \wedge U_{<s} \beta\right) \rightarrow U_{<t+s}(\gamma \vee \beta), t+s \leq 1$.

Proof of (a). Recall that the formula

$$
\left(U_{\geq t} \gamma \wedge U_{\geq s} \beta \wedge U_{\geq 1}(\neg \gamma \vee \neg \beta)\right) \rightarrow U_{\geq \min \{1, t+s\}}(\gamma \vee \beta)
$$

can be written as:

$$
\left(U_{\leq 1-t} \neg \gamma \wedge U_{\leq 1-s} \neg \beta \wedge U_{\leq 0}(\gamma \wedge \beta)\right) \rightarrow U_{\leq 1-\min \{1, t+s\}} \neg(\gamma \vee \beta)
$$

Now, consider the axiom A5 for:

$m=3 ; n=k=1 ; r_{1}=1-t ; r_{2}=1-s ; r_{3}=0 ;$

$\alpha_{1}=\neg \gamma ; \quad \alpha_{2}=\neg \beta ; \quad \alpha_{3}=\gamma \wedge \beta ; \quad \alpha=\neg(\gamma \vee \beta)$.

We obtain that $r=1-(t+s)$.

(i) If $t+s>1$ then (Axiom A6, $\sum_{i=1}^{m} r_{i}<k$ )

$$
\vdash \neg\left(U_{\leq 1-t} \neg \gamma \wedge U_{\leq 1-s} \neg \beta \wedge U_{\leq 0}(\gamma \wedge \beta)\right)
$$

and hence

$$
\left.\vdash\left(U_{\leq 1-t} \neg \gamma \wedge U_{\leq 1-s} \neg \beta \wedge U_{\leq 0}(\gamma \wedge \beta)\right) \rightarrow U_{\leq 1-\min \{1, t+s\}} \neg(\gamma \vee \beta)\right) .
$$


(ii) If $t+s \leq 1$, then $1-\min \{1, t+s\}=1-(t+s)=r$ and it is left to check if

$$
\alpha \rightarrow \bigvee_{J \subseteq\{1,2,3\},|J|=2} \bigwedge_{j \in J} \alpha_{j}
$$

and

$$
\bigvee_{J \subseteq\{1,2,3\},|J|=1} \bigwedge_{j \in J} \alpha_{j}
$$

are tautologies. Namely, in this case, the first formula has the following form: $\neg(\gamma \vee \beta) \rightarrow((\neg \gamma \wedge \neg \beta) \vee(\neg \gamma \wedge \gamma \wedge \beta) \vee(\neg \beta \wedge \gamma \wedge \beta))$, and the second formula: $\neg \gamma \vee \neg \beta \vee(\gamma \wedge \beta)$. It is obvious that both of these formulas are tautologies and therefore is this part proved.

Proof of (b).

Let us show equivalently that ILUPP ${ }^{E x t} \vdash\left(U_{\leq t} \gamma \wedge U_{\geq t+s}(\gamma \vee \beta)\right) \rightarrow U_{\geq s} \beta$ :

$\vdash U_{\geq t+s}(\gamma \vee \beta) \rightarrow U_{>t+s^{\prime}}(\gamma \vee \beta)$, for all $s^{\prime}<s \quad$ (contraposition of A3)

$U_{\leq t} \gamma \wedge U_{\geq t+s}(\gamma \vee \beta) \vdash U_{\leq t} \gamma \wedge U_{>t+s^{\prime}}(\gamma \vee \beta)$, for all $s^{\prime}<s$

$U_{\leq t} \gamma \wedge U_{\geq t+s}(\gamma \vee \beta) \vdash U_{\leq t} \gamma \wedge U_{>s^{\prime}} \beta$, for all $s^{\prime}<s \quad$ (by Lemma 4)

$U_{\leq t} \gamma \wedge U_{\geq t+s}(\gamma \vee \beta) \vdash U_{\geq s} \beta \quad$ (by Inference Rule (3))

$\vdash\left(U_{\leq t} \gamma \wedge U_{\geq t+s}(\gamma \vee \beta)\right) \rightarrow U_{\geq s} \beta \quad$ (by Deduction theorem)

\section{Conclusion}

In this paper we present the proof-theoretical analysis of two logics which allow making statements about upper and lower probabilities. The introduced formalisms, the propositional logic ILUPP and its first-order extension ILUPFO, can be used for reasoning not only about lower and upper probabilities an agent assigns to a certain event, but also about her uncertain belief about other agent's imprecise probabilities. The languages of our logics are modal languages which extend classical propositional/first-order languages with the unary operators $U_{\geq r}^{a}$ and $L_{\geq r}^{a}$, where $a$ is an agent and $r$ ranges over the unit interval of rational numbers. The corresponding semantics consist of the measurable Kripke models with sets of finitely additive probability measures attached to each possible world.

We prove that the proposed axiomatic systems are strongly complete with respect to the class of measurable models. Since the logics are not compact, the axiomatizations contain infinitary rules of inference. In [26] it is shown that the same axiomatic systems (the only difference is that in [26] only one agent is considered) is sound and complete for the logics without nesting of probabilistic operators. This situation is not an exception. For example, modal system $K$ is sound and complete with respect to the class of all modal models, but also with respect to the class of all irreflexive models [14].

We provided the extensions of the proposed axiomatizations in order to properly capture the notions of lower and upper probability of an infinite set of agents. We also showed that the logics ILUPP and ILUPFO generalize the logics for reasoning about sharp probabilities from [23,24]. 
We also prove that the satisfiability problem for ILUPP logic is decidable. In the proof, we use the method of filtration [14] to show that if a formula is satisfiable in a world $w$ of an ILUPP structure, then it is satisfiable in a finite structure. We also use a reduction to linear programming to deal with infinitely many probability measures definable on finite algebras, and to solve the satisfiability problem in a finite number of steps.

We propose two topics for future work. First, we would like to investigate an alternative to the approach of upper and lower probability of a group presented here. We assumed that the agents share their sets of probabilities in order to obtain a larger set, available to all the members of the group. Alternatively, we wish to investigate the scenario in which they share their constraints, i.e., a group accepts those probability measures which satisfy lower and upper constraints of all the members of the group (i.e., $L_{\geq s}^{G} \alpha$ would be defined as a disjunction, and $U_{\geq s}^{G} \alpha$ as a conjunction). Note that this approach leads to an debatable consequence: consider the group $G=\{a, b\}$, where both lower and upper probability of $\alpha$ are $\frac{1}{2}$ for the agent $a$, while they are both $\frac{1}{3}$ for $b$; then lower probability of $\alpha$ wrt. $G$ would be greater then the corresponding upper probability. This indicates that this approach is sensible if the formulas capture agents' imprecise knowledge about an objective probability, in which case there shouldn't be inconsistencies between the knowledge of different agents. In terms of semantics, the upper and lower probabilities of different agents should be all satisfied by a probability measure.

Second, we will investigate complexity of satisfiability problem for the logic ILUPP. Such a method is already developed in [16] for probabilistic logics with iterations of standard probability operators. Note that the fact that ILUPP is a generalization of the logic LPP1 already leads to a complexity bound for ILUPP. Namely, it was shown in [16] that the satisfiability problem for the logic LPP1 is PSPACE-complete, thus a lower complexity bound for ILUPP is PSPACE.

\section{References}

1. B. Anger, J. Lembcke. Infinitely subadditive capacities as upper envelopes of measures. Zeitschrift fur Wahrscheinlichkeitstheorie und Verwandte Gebiete, 68: 403-414. 1985.

2. G. de Cooman, F. Hermans. Imprecise probability trees: Bridging two theories of imprecise probability. Artificial Intelligence 172(11): 1400-1427. 2008.

3. D. Doder, N. Savić, Z. Ognjanović. A Decidable Multi-agent Logic with Iterations of Upper and Lower Probability Operators. In: Proc. of FoIKS, Lecture Notes in Computer Science, Springer 170-185. 2018

4. D. Doder, Z . Marković, Z. Ognjanović, A. Perović, M. Rašković. A probabilistic temporal logic that can model reasoning about evidence. In: Proc. of FoIKS, Lecture Notes in Computer Science, Springer 9-24. 2010

5. D. Dubois, H. Prade. Possibility Theory. Plenum Press, New York, 1988.

6. R. Fagin, J. Halpern, N. Megiddo. A logic for reasoning about probabilities. Information and Computation 87(1-2):78-128. 1990.

7. R. Fagin, J. Halpern. Reasoning about knowledge and probability. Journal of the ACM, 41(2): 340-367, 1994.

8. M. Fattorosi-Barnaba, G. Amati. Modal operators with probabilistic interpretations I. Studia Logica 46(4): 383-393. 1989. 
9. A. Frish, P. Haddawy. Anytime deduction for probabilistic logic. Artificial Intelligence 69: 93-122. 1994.

10. J. Y. Halpern. An analysis of first-order logics of probability. Artificial Intelligence 46: 311-350. 1990.

11. J. Y. Halpern, R. Pucella. A Logic for Reasoning about Upper Probabilities. Journal of Artificial Intelligence Research 17: 57-81. 2002.

12. J. Halpern, R. Shore. Reasoning about common knowledge with infinitely many agents. Information and Computation 191:1-40. 2004.

13. P.J. Huber. Robust Statistics. Wiley, New York, 1981.

14. G.E. Hughes, M.J. Cresswell. A companion to modal logic, Methuen, 1984.

15. A. Heifetz, P. Mongin. Probability logic for type spaces. Games and economic behavior 35: 31-53. 2001.

16. I. Kokkinis. The Complexity of Satisfiability in Non-Iterated and Iterated Probabilistic Logics, arXiv:1712.00810v1, 2017.

17. H.E. Kyburg. Probability and the Logic of Rational Belief. Wesleyan University Press, Middletown, Connecticut, 1961.

18. I. Levi. The Enterprise of Knowledge. MIT Press, London, 1980.

19. G.G. Lorentz. Multiply subadditive functions. Canadian Journal of Mathematics 4(4): 455-462. 1952

20. M. Meier. An infinitary probability logic for type spaces. Israel Journal of Mathematics 192(1): 1-58. 2012.

21. E. Miranda. A survey of the theory of coherent lower previsions. International Journal of Approximate Reasoning vol. 48, no. 2: 628-658. 2008.

22. Z. Ognjanović, M. Rašković. Some probability logics with new types of probability operators. Journal of Logic and Computation 9(2): 181-195. 1999.

23. Z. Ognjanović, M. Rašković. Some first-order probability logics. Theoretical Computer Science 247(1-2): 191-212. 2000.

24. Z. Ognjanović, M. Rasković, Z. Marković. 2016. Probability Logics - Probability-Based Formalization of Uncertain Reasoning. Springer.

25. M. Rašković, Z. Marković, Z. Ognjanović. A logic with approximate conditional probabilities that can model default reasoning. International Journal of Approximate Reasoning vol. 49, no. 1: 52-66. 2008.

26. N. Savić, D. Doder, Z. Ognjanović. Logics with Lower and Upper Probability Operators. International Journal of Approximate Reasoning, 88: 148-168. 2017.

27. N. Savić, D. Doder, Z. Ognjanović. A First-Order Logic for Reasoning About HigherOrder Upper and Lower Probabilities. In: Proc. of ECSQARU, Lecture Notes in Artificial intelligence, Springer 491-500. 2017.

28. G. Shafer. A Mathematical Theory of Evidence. Princeton University Press, Princeton, NJ, 1976.

29. S. Tomović, Z. Ognjanović, D. Doder. Probabilistic common knowledge among infinite number of agents.. In: Proc. of ECSQARU, Lecture Notes in Artificial intelligence, Springer 496-505. 2015

30. P. Walley. Statistical Reasoning with Imprecise Probabilities. Chapman and Hall, London, 1991.

31. P. Walley. Towards a unified theory of imprecise probability. International Journal of Approximate Reasoning vol. 24, no. 2-3: 125-148. 2000.

32. W. van der Hoek. Some consideration on the logics $P_{F} D$. Journal of Applied NonClassical Logics, vol. 7, no. 3, 287 - 307. 1997.

33. L.A. Zadeh. Fuzzy sets as a basis for a theory of possibility. Fuzzy Sets and Systems 1: 3-28. 1978. 